\title{
VIRTUAL GROUPS 45 YEARS LATER
}

\author{
by Calvin C. Moore \\ Dedicated to the memory of George W. Mackey
}

\begin{abstract}
In 1961 George Mackey introduced the concept of a virtual group as an equivalence class under similarity of ergodic measured groupoids, and he developed this circle of ideas in subsequent papers in 1963 and 1966. The goal here is first to explain these ideas, place them in a larger context, and then to show how they have influenced and helped to shape developments in four different but related areas of research over the following 45 years. These areas include first the general area and the connections between ergodic group actions, von Neumann algebras, measurable group theory and rigidity theorems. Then we turn to the second area concerning topological groupoids, $C^{*}$-algebras, $K$-theory and cyclic homology, or as it is now termed non-commutative geometry. We briefly discuss some aspects of Lie groupoids, and finally we shall turn attention to the fourth area of Borel equivalence relations seen as a part of descriptive set theory. In each case we trace the influence that Mackey's ideas have had in shaping each of these four areas of research.
\end{abstract}

1. Introduction. In his 1961 American Mathematical Society Colloquium Lectures, George Mackey introduced his concept of a virtual group. He briefly described this on pages 651-654 in his Bulletin article [Ma63a] that was based on his Colloquium lectures, and then discussed it again in a research announcement in the Proceedings of the National Academy of Science [Ma63b] and then more fully in [Ma66]. His concept of virtual groups was also a theme in some of his subsequent integrative and expository articles and books. The basic motivation for this notion comes from the study of actions of a second countable locally compact group $G$ (and subsequently all locally compact groups will be assumed to be second countable without further mention) on a "good" Borel space $X$, which is a Borel action in an obvious sense. It further assumed that there is a measure $\mu$ on $X$ which is quasi-invariant under the action of $G$ in that the measure class of $\mu$ is invariant. A special case of great importance is when there is an invariant probability measure, but such a hypothesis is too constraining in general. Finally it is assumed that the action of $G$ on $(X, \mu)$ is ergodic in the sense that any $G$ invariant set is either a null set or a co-null set for $\mu$. Such ergodic actions will be 
subjected to a new notion of equivalence or similarity, which amounts to what is called orbit equivalence, and which we will describe shortly. This notion of similarity will be the key to the definition of a virtual group.

The prime example of an ergodic action of $G$ is formed by taking a closed subgroup $H$ of $G$ and letting $G$ act on the coset space $H \backslash G$, where we are letting $G$ act on the right as this works out better for notational purposes. The coset space has a unique equivalence class of quasi-invariant measures on it, and action is clearly ergodic - in fact it is transitive and the only invariant sets are the null set and the whole space. Transitive actions are well behaved in many senses while the non-transitive ergodic actions (sometimes called properly ergodic actions) present many difficult and complex problems. In fact, in the very early days of statistical mechanics, the subject from which ergodic theory arose, no one saw how to achieve the fundamental goal of justifying the equating of phase and time averages except in the case of a transitive time action (which was a topological impossibility). It was only in 1931 when Birkoff and von Neumann published their respective and complementary ergodic theorems that ergodic theory as we know it was established.

A transitive action of $G$ is defined completely by the isotropy subgroup $H$, or perhaps more properly by the conjugacy class of the subgroup $H$. The notion of similarity or equivalence of ergodic actions that we will introduce shortly makes the action of $G$ on $H \backslash G$ similar to the action of $H$ on a point so that up to similarity the action of $G$ on $H \backslash G$ is a subgroup (or a conjugacy class of subgroups) of $G$. Mackey's fundamental idea was to view a properly ergodic action of $G$ on some space $X$ ( or rather its similarity class) as a kind of generalized subgroup of $G$ - or as he called it, a virtual group. As such one will be able to speak of unitary representations of these objects; induced representations are well defined and there is a version of the imprimitivity theorem for them. Operator algebras - both von Neumann algebras and in some cases $C^{*}$-algebras can be associated with these objects just as for ordinary locally compact groups.

Mackey's hope was that this point of view would shed light on the difficulties and complexities of non-transitive ergodic actions. Indeed, one can argue that this program introduced by Mackey was an opening shot in what would develop as non-commutative geometry and topology. Sections 5, 6, and 7 of [Ma63b] very clearly adumbrate future developments along these lines. The goal of this paper is to give a brief and necessarily selective account of some of the developments that have taken place over the 45 years since Mackey introduced these ideas, and which can be traced back to him and his work. Hence the title of this paper-Virtual Groups 45 Years Later.

2. Groupoids. It is now time to introduce the formal definition, and for this we need the concept of a groupoid. A groupoid is a set $G$ with a partially defined associative multiplication such that every element has an inverse with $g \cdot g^{-1}$ and $g^{-1} \cdot g$ being left and right units for $g$. Equivalently, one could say that a groupoid is a small category (i.e., a set) with the property that all morphisms are invertible. Some may find this definition in terms of 
categories quite elegant, but others might find it a little too slick. In any case there is a set of units in $G$, (the elements such that $x \cdot x=x$ where $x \cdot y$ is the multiplication in $G$ ) which we will write as $X$, and which is sometimes view as a subset of $G$ but also sometimes viewed as a separate set. A groupoid always comes with two maps from $G$ to $X$ - the range map $r$ and source map $s$ where $r(g)$ is the left unit for $g$ and $s(g)$ is the right unit for $g$. The definition of a homomorphism of one groupoid to another is self evident - a map $f$ from one groupoid to another such that if the product $x \cdot y$ is defined, then $f(x) \cdot f(y)$ is defined and is equal to $f(x \cdot y)$.

The concept of groupoid is best developed by a sequence of examples. The first and most obvious example is that of a group $G$. The unit space is one element. A second example consists of a set $X$ together with field of groups $G_{x}$, one for each point of $X$. One thinks of the groups $G_{x}$ as stalks lying over the point $x$ in $X$, and the groupoid is the union of the groups $G_{x}$. Groupoid multiplication is defined for two elements iff they are in the same stalk and the groupoid product is the group product in the stalk. The set $X$, viewed as the units in the union of the $G_{x}$, is the set of units in the groupoid. A third and very important example is an equivalence relation $R$ on a set $X$ with $R$ is a subset of $X \times X$. The set $R$ is the groupoid, $X$ is the set of units, and the product $(x, y) \cdot(w, z)$ is defined exactly when $y=w$, and then the product is $(x, z)$. The inverse of $(x, y)$ is $(y \cdot x)$, and the left and right units of $(x, y)$ are respectively $(x, x)$ and $(y, y)$ so $r((x, y))=(x, x)$ and $s((x, y))=(y, y)$. One may think of the units of $R$ as either $X$ itself or as the diagonal $\Delta X$ contained in $R$.

Any groupoid $G$ defines an equivalence relation $R$ on its set of units by defining $(x, y) \in R$ if and only if there is a $g$ in $G$ such that $g \cdot g^{-1}=x$ and $g^{-1} \cdot g=y$. The defining properties of a groupoid ensure that this is a equivalence relation, and then the map from $G$ to $X \times X$ defined by $f(g)=(r(g), s(g))$ is a homomorphism of groupoids. The inverse image under $f$ of a point $(x, x)$ in $G$ is readily seen to be a group, which we call the isotropy or holonomy group $G_{x}$ at $x$. A groupoid is called principal if it is equal to the corresponding equivalence relation, or equivalently if the $G_{x}$ are all trivial. In general the homomorphism of a groupoid to its principal groupoid is onto and can be viewed as having as its kernel the groupoid consisting of the field of groups $G_{x}$ over the set $X$. Of course there are also the sets $G_{x, y}$, the inverse image under $F$ of the point $(x, y)$, where we have abbreviated $G_{x, x}$ as $G_{x}$.

A group $H$ acting on a set $X$ also defines a groupoid. The groupoid $G$ is $X \times H$ and the set of units is $X$. The product $(x, g) \cdot(y, h)$ is defined exactly when $x \cdot g=y$ (where for convenience of notation, we let $H$ operate on the right on $X)$ and the product is $(x, g h)$. The equivalence relation on $X$ is the equivalence relation of being in the same $H$-orbit, and the groups $G_{x}$ are just the isotropy subgroups $H_{x}$ of the action of $H$ on $X$. It should be remarked that when we go from an action of a group $H$ on a set $X$ to the groupoid $G$, generally much of the original group $H$ disappears. The only part of $H$ left are the isotropy subgroups $H_{x}$ (and perhaps also the sets $G_{x, y}$, which are the elements of $H$ taking $y$ to $x$ ). If the action of $H$ is free, then $H$ disappears completely in the groupoid picture. At the other extreme, if $X$ is a point, the groupoid is just the group $H$. 
A final example anticipates later sections of the article, but is useful to mention now. If $X$ is a foliated manifold (or a foliated space - [MoSc06]), then one can define an equivalence relation on $X$ by making the equivalence classes the leaves of the foliation. This produces a principal groupoid, but the geometry virtually forces a more subtle construction where one adds in the holonomy group of each point (or leaf) and constructs the holonomy groupoid $G$ of the foliation [Wi83]. The principal groupoid associated to $G$ is the equivalence relation given by the leaves and the group $G_{x}$ is exactly the holonomy groups of the foliation at $x$. In general the equivalence relation cannot be given the structure of a manifold, but the holonomy groupiod can be given the structure of a (locally Hausdorff) manifold. See [Wi83], [CC00] and [CC03] for more details.

A construction that is very natural from the point of view of groupoids is that of restriction. Namely if $Y$ is a subset of the unit space of a groupoid $G$, one can form a new groupoid $G_{Y}$, which is defined to be the inverse image in $G$ of $Y \times Y$ by the map $f(g)=(s(g), r(g))$. If the groupoid is principal - an equivalence relation $R$, then $R_{Y}$ is just $R \cap Y \times Y$. If $G$ came from a group action on $X$, this operation leaves one with no trace of a group action. On the other hand if $G$ is the groupoid of a foliation, and if for instance $Y$ is a subset or a submanifold of $Y$, in particular if $Y$ is a transversal (set meeting each leaf in a countable set), then $G_{Y}$ is a very natural and important object. The notion of the product of two groupoids $G_{1}$ and $G_{2}$ is a natural one for one one just takes the product of the two groupoids. The unit space of the product is the product of the unit spaces.

Just as one can define what it means for an abelian group $A$ to be a module for a group $G$-there is homomorphism of $G$ into the automorphism group of $A$-we can similarly define a module for a groupoid $G$. Suppose that $X$ is a set and $R$ an equivalence relation on $X$, and that $A_{x}$ is a field of abelian groups on $X$ such that $A_{x}$ and $A_{y}$ are isomorphic if $x$ and $y$ are equivalent under $R$. An automorphism of such a structure is the giving for each pair $(x, y)$ in $R$ of an isomorphism of $A_{y}$ onto $A_{x}$, with the usual multiplication property for triples $(x, y, z)$. The set of such automorphisms is a groupoid with unit space $X$ and equivalence relation $R$. Then if $G$ is a groupoid with unit space $X$ and equivalence relation $R$, such a field of abelian groups is called a module for $G$ if there is given homomorphism of groupoids over $X$ from $G$ into the automorphism groupoid of the field of groups. A special case of interest is when the field $A_{x}$ is a constant field and the isomorphism from $A$ to $A$ corresponding to any pair $(x, y)$ is the identity. If $A$ is now a Hilbert space we have the notion of a unitary representation of the groupoid.

Whenever $A$ is module for a groupoid, one can define just as in the group case, cohomology groups of the pair $G, A$ where the definition of an $n$-cocyle virtually makes itself. When the groupoids have additional structure that we will subsequently describe, various supplemental conditions on cocycles will naturally occur, and we shall meet these on several occasions subsequently. Also for $n=1$, one can allow a non-commutative group as the module, and such 1-cocycles will play a major role as the theory develops. They are in fact just homomorphisms from the groupoid $G$ to the coefficient group $A$. 
Similarly if $G$ is a groupoid one can define the notion of a $G$-space $S$ so that $G$ acts on $S$ as transformation groupoid on $S$, just as a group can act as transformation group on a set. If $G$ is a groupoid with unit space $X$, a $G$-space is a set $S$ which is fibered over $X$ by a map $\pi$ and we are given a groupoid homomorphism from $G$ into partially defined maps of $S$ to itself with the action of acting with $g$ in $G$ on an element of $S$ written as $s \cdot g$, so that $s \cdot g$ is defined when $s(g)=\pi(s)$ and then $\pi(s \cdot g)=r(g)$.

One is always a little suspicious of new classes of mathematical objects obtained by weakening the axioms of traditional objects, but the notion of a groupoid has proven to be a surprisingly useful and effective one in many areas of mathematics - see for instance the entire volume [RaRe01]. Some have argued that ultimately the basic concept involved here will be seen to be that of a groupoid, and that groups will emerge as just a special case of groupoids [Br87]. However, a somewhat more productive view is to see groupoids more as equivalence relations with additional structure - that is, a field of holonomy groups, rather than as generalizations of groups.

The goal in the following is to trace some of the developments since the 1960's of the circle of ideas that Mackey introduced about ergodic theory, groupoids, and what he called virtual groups. We will explore in other words the intellectual heritage that we received from Mackey and his work in this area. One common theme will be to see how properties that we normally think of as properties of groups also make sense in certain contexts for groupoids and equivalence relations, such as amenability and Kazhdan's Property $(T)$. A related theme is the extent to which an equivalence relation generated by a free action of group will encode within its structure properties or information about the group. Both of these themes reflect Mackey's view that equivalence relations and groupoids have more in common with groups than may be evident at first site, and which justify the term "virtual group".

We will trace developments along four different avenues or categories of objects. The first is the original category Mackey proposed of ergodic measured groupoids, which will lead us to many interesting questions about von Neumann algebras that can be constructed from measured groupoids, and also to many interesting questions about geometric group theory, rigidity, and to what can be called measurable group theory-see [Sh05]. Second we will briefly examine the topological category consisting of locally compact topological groupoids; Renault [Ren80] outlined the connection between these topological groupoids and $C^{*}$-algebras, a connection that is analogous to the relationship of measured groupoids to von Neumann algebras. Alain Connes and many others have developed this line of work into a powerful and beautiful edifice with connections to foliations, index theory, and questions in topology such as the Novikov conjecture. One key question is how to calculate and use in applications the $K$-theory groups of the $C^{*}$-algebras associated with topological groupoids. The third avenue that we will explore, and that only briefly will be that of smooth or Lie groupoids, where the main approach will be as special case of topological groupoids. There is of course a vast literature on smooth or Lie groupoids and its connections with differential 
geometry that we will not explore - see [Mack87], [Mack05], [Wein87], and [Wein92]. Finally we will briefly survey a fourth avenue of research in which groupoids are studied in the Borel category. That is one considers a Borel groupoid $G$ where $G$ and its unit space are standard Borel spaces, with no measures, and with results holding everywhere, not just almost everywhere. As such, the theory becomes in effect a very interesting and new part of classical descriptive set theory. Here the focus will be on equivalence relations and indeed only those with countable orbits. Alexander Kechris and Greg Hjorth and many others have developed this theory into a rich and fascinating subject and results obtained in this context feed back and enrich the other avenues that we will explore. The paper includes a substantial list of references to the literature.

3. Measured Groupoids. Using this discussion of groupoids as abstract structures, let us now introduce the additional analytic structure that will be needed for our discussion. We first assume that the groupoid $G$ has Borel structure in which it is good Borel space- usually assumed to be standard Borel space, but analytic Borel space will also suffice, and likewise for the unit space $X$ - see [Ma57] for this terminology. All maps defining the groupoids are assumed to be Borel, and so we have a Borel groupoid. Then we assume first that there is a measure $\mu$ on $G$, whose measure class is invariant under the map $g \rightarrow g^{-1}$. That condition insures that the images of $\mu$ on under $r$ and $s$ are equivalent - we call one of them $\nu$ and then we require that when $\mu$ is disintegrated over $X$, the fiber measures satisfy an almost everywhere invariance condition. See [Ram71] for details, and also very imporantly see [Hah78a] for how to generate what is called a Haar measure. These data give us a measured groupoid, and the remaining condition we need is ergodicity. First we say that a Borel subset $Y$ of the unit space $X$ of $G$ is invariant if $r^{-1}(Y)=s^{-1}(Y)$, and ergodicity means that any invariant Borel set is null or conull for $\nu$. The product of two measured groupoids is again a measured groupoid and will be ergodic if both factors are ergodic.

In dealing with measured groupoids, it is convenient and indeed essential to at times replace a groupoid by an inessential contraction, which is simply $G_{S}$ (see above) where $S$ is a $\nu$ co-null set of units. The notion of an isomorphism of measured groupoids is clear - we have a Borel map from one to other that is multiplicative almost everywhere and invertible up to null set which takes measure classes to measure classes - see [Ram71]. A measured groupoid $G$ is isomorphic to $G_{Y}$ where $Y$ is $\nu$ co-null. The notion of homomorphism and the all important notion of similarity or equivalence are in fact a bit more subtle and technical and are discussed in [Ram71]. We will a take slightly different approach to similarity. We let $\mathscr{I}$ be the principal groupoid or equivalence relation whose unit space is $I$ the unit interval with all points equivalent so $\mathscr{I}$ is $I \times I$. We put Lebesgue measure on $I$ and the product measure on $I \times I$, and $\mathscr{I}$ is a measure groupoid and in fact an ergodic one. Then we say that two measure groupoids $G_{1}$ and $G_{2}$ are similar (or equivalent or stably isomorphic) if $G_{1} \times \mathscr{I}$ is isomorphic to $G_{2} \times \mathscr{I}$.

The basic idea here is that two measured groupoids are similar or equivalent if when we 
"fatten up" the orbits in each of them by crossing each orbits with the unit interval carrying Lebesgue measure, then the measured groupoids become isomorphic. That this is the same as similarity as for instance defined in [Ram71] is clear from Theorem 6.1 of [Ram82] and [FHM78]. Moreover, similarity of two measure groupoids $G_{1}$ and $G_{2}$ effectively means that the orbit space $X_{1} / R_{1}$ (the quotient of of the unit space mod the equivalence relation) is the same up to null sets as the orbit space for the other $X_{2} / R_{2}$, and of course that the holonomy groups of corresponding orbits for the two groupoids are the same. In the ergodic case, Alain Connes has called these orbit spaces the "little spaces" for if $G$ is ergodic and $Y$ is any subset of the unit space $X$ of $G$ of positive measure no matter how small, then $G_{Y}$ is similar to $G$. As we have formulated the notion of similarity or equivalence, it is perhaps more suitably called stable isomorphism, a term that we will use frequently. When we examine properties and constructions involving measured groupoids, we will need to pay attention to whether they are invariant under similarity - that is, stable isomorphism.

Finally, Mackey defines a virtual group as a similarity class of ergodic measured groupoids, or a stable isomorphism class of such. The rationale for this terminology "virtual group" comes from the transitive case where the measured groupoid $G$ is generated by the action of a locally compact group $H$ on a coset space $K \backslash H$ of $H$, where $K \backslash H$ carries the unique $H$ quasi-invariant measure class. It is perfectly clear from the definition of similarity that this groupoid is similar to the group $K$ (operating on a point) or just to the group $K$, which is a subgroup of $H$. Mackey's point in 1961 was that a properly ergodic (non-transitive) measured groupoid resembled in many ways a real subgroup of $H$, and that this point of view and analogy with subgroups might help in understanding properly ergodic actions, especially in the context of analyzing representations of group extensions-Mackey's little group method [Ma58]. What this approach does in addition is to begin to visualize the space of orbits of such groupoids as a geometrical object, just as one views the space of leaves of a foliation as a geometric object similar to and generalizing the base space of a fibre bundle when the bundle is viewed as a special case of a foliation. This is the sense in which Mackey's idea was an opening shot in non-commutative geometry and topology.

Similarly, as in the case of abstract groupoids one can define what one means by a Borel $G$ space of a groupoid - one has a Borel space $S$ fibered over the unit space of $G$ and $G$ acting as partially defined maps of $S$ to itself just as above for abstract groupoids. One can what is means for a measure on $S$ to be quasi-invariant and so one has the notion of a measured $G$ space $S$.

Again as in the case for abstract groupoids, one can define a module for a Borel or measured groupoid $G$ as a measurable field $A$ of Polish (that is, admitting the structure of a complete and separable metric space) abelian groups over the unit space where the action is a Borel map. In case $A$ is a separable Hilbert space and automorphisms are regarded as unitary operators, we have the notion of a unitary representation of measured groupoid. Now it is easy to see how to start with a module $A$ for measured groupoid $G$ and turn it into one for the groupoid $G \times \mathscr{I}$, and conversely one can start with a module for $G \times \mathscr{I}$ and find 
a corresponding one for $G$. This in effect a (rarefied) version of the Mackey imprimitivity theorem. As special case one see that there is natural equivalence between classes of unitary representation of stably isomorphic measured groupoids. In fact the pairing up of unitary representations of the groupoid $G$ generated by the action of a group $H$ on a coset space $K \backslash H$ of itself with unitary representations of $K$ is itself Mackey's original imprimitivity theorem [Ma49, Ma51]. One can develop a theory of unitary representations of measured groupoids but we shall not go any farther in that direction here

One can define cohomology groups $H^{*}(G, A)$ either axiomatically of by one of two kinds of cocyles complexes. It turns out just as in the case of locally compact groups that it does not matter whether one uses cocyles that are defined everywhere and satisfy the cocycle identity everywhere and identify cocycles which agree off of appropriate conull sets or whether one uses measurable cocycles that satisfy the cocycle identity almost everywhere - [Wes69], [Wes71], [FM77], [FHM78], [Ser81]. If $G$ and $K$ are stably isomorphic and $A$ and $B$ are corresponding $G$ and $H$ modules under this stable isomorphism, then the cohomology groups $H^{*}(G, A)$ and $H^{*}(K, B)$ are isomorphic [Ser81]. This is a thoroughly disguised version of Shapiro's Lemma for finite group cohomology. As before for dimension 1, we can allow noncommutative modules and 1-cocycles are simply groupoid homorphisms from $G$ either to a the groupoid $G$ to the groupoid consisting of the field of coefficient groups or in the event of a constant field consisting of a single group $H$, then it is a groupoid homomorphism to the group $H$.

Every measured groupoid has a very important one-cocyle or homomorphism to the multiplicative group of the positive real numbers - the Radon-Nikodym cocycle. One first has to arrange the measure $\mu$ on $G$ to have certain invariance properties so that it is what Hahn [Hah78a] calls a Haar measure, and then the Radon-Nikodym derivative of $\mu$ with respect to its transform by the inversion map yields a groupoid homomorphism to $R^{*+}$ [Hah78a]. This is exactly parallel to the group case where the modular function is an ordinary homomorphism to $R^{*+}$. Different choices of a Haar measure yield by a simple calculation cohomologous cocycles or homomorphisms so this construction yields a unique cohomology class.

Mackey observed that if one has a homomorphism $\phi$ from an ergodic measured groupoid $G$ to a group $H$, the range of the homorphism - whatever that is - should be a virtual subgroup of $H$, or in other words should define an ergodic action of $H$ on some measure space. Mackey shows how to do this [Ma66]. One first forms the Borel space $H \times G$ and define a map to $X$, the unit space of $G$ by $\pi\left(\left(h, g^{\prime}\right)\right)=r\left(g^{\prime}\right)$ and let $G$ act on this fibered space by $\left(h, g^{\prime}\right) \cdot g=$ $\left(h \phi(g)^{-1}, g^{\prime} g\right)$ when $s(g)=\pi\left(\left(h, g^{\prime}\right)\right)=r\left(g^{\prime}\right)$. The measure on $H \times G$ is Haar measure on $G$ times the measure on $G$. This action is no longer ergodic, but does have a decomposition into ergodic pieces indexed by a Borel space $Y$. Since the action of $G$ on $H \times G$ commutes with the action of $H$ on the left on the first factor, $H$ permutes the ergodic components and this is a Borel action of $H$ on $Y$ together with a quasi-invariant measure. Since $G$ was ergodic, it follows that $H$ is ergodic on $Y$ and so $Y \times H$ becomes an ergodic measured groupoid. This 
is called the Mackey range of the homomorphism $\phi$. Chasing through the definition, one sees that cohomologous homomorphisms define isomorphic Mackey ranges. See [Ma66] for details. In the case of the Radon-Nikodym cocycle or homorphism, the corresponding flow of the real line (really $R^{*+}$ ) is called the Radon-Nikodym flow, and it is an object that will be of key importance in the following section on hyperfinite groupoids.

If the ergodic measured groupoid $G$ is a transitive one defined by the action of a locally compact group $K$ on a coset space $L \backslash K$ of itself, a homorphism of $G=L \backslash K \times K$ to a locally compact group $H$ simply amounts to a homomorphism $\phi$ of $L$ into $H$, and again by chasing through the definitions, one sees that the Mackey range of $\phi$ is the transitive action of $H$ on its coset space $H$ mod the closure of the range of $\phi,(\phi(L))^{-} \backslash H$ so the definition in this case matches up with one expects of the range (or rather the closure of the range). Of course one can do the same thing construction for a homomorphism of one ergodic groupoid to another, but the details are too mind-numbing to include here.

4. Von Neumann Algebras, Discreteness, and Hyperfiniteness. For any measured groupoid $G$ it is possible to construct a von Neumann algebra. This construction for group measure space action is quite venerable and goes back to the very founding of von Neumann algebras in the 1930's. This is also a well-known construction for any locally compact group as the enveloping algebra of the regular representation. The construction was crucial in the work of Araki and Woods, Krieger, and Connes as described below. In the completely general case of measured groupoids, Hahn [Hah78b] showed how to overcome certain measure theoretic complexities not present in the discrete case and carry out the construction. Given the richness, diversity and ubiquity of the examples of groupoid von Neumann algebras, it is perhaps not too much of an overstatement to say that all interesting examples of von Neumann algebras come from this construction.

In the special case of measured groupoids where the map $r$ (or equivalently the map $s$ ) has countable fibers, many of the messy and technical considerations about homomorphisms and similarities, which we have largely swept under the rug here, become immensely simpler. We shall call such measured groupoids discrete, and for the remainder of this survey, we will largely concentrate on this special case. The discreteness condition means that all holonomy groups are countable and that all orbits of the equivalence relation are countable. This will always be the case for the groupoid generated by an action of a countable group or for the groupoid of a foliation relativized to a transversal. In a sense this is already almost the general case, for by a result of Ramsay [Ram82], every measured groupoid with countable holonomy groups is stably isomorphic to a discrete measured groupoid, and so in particular every measured equivalence relation is similar (or stably isomorphic) to one with countable orbits.

Within the class of discrete measured groupoids, the notion of similarity or stable orbit equivalence can be so simplified. We let $I_{\infty}$ be the measured groupoid (equivalence relation) which is $Z \times Z$ with unit space $Z$ and all points equivalent and with counting measure. Then 
it is clear that two discrete measured groupoids $G_{1}$ and $G_{2}$ are similar or stably isomorphic if and only if $G_{1} \times I_{\infty}$ is isomorphic to $G_{2} \times I_{\infty}$. If one starts with a discrete groupoid $G$ with unit space $X$, then for any measure $\nu$ on $X$, we can build a measure $\mu$ on $G$ say by using the counting measure on the fibers of $s$ and integrating these over $\nu$ to get $\mu$. Then $\nu$ will be quasi-invariant under $G$ exactly when the class of $\mu$ is invariant under the flip $g \rightarrow g^{-1}$ on $G$. Then $r$ and $s$ map Borel sets to Borel sets and the saturation of a Borel set in $X$ is again a Borel set. Finally it was shown in [FM77] that a discrete measured equivalence relation was always generated by the action of a countable group. It was left open whether one could find a free action of a countable group that generated the equivalence relation. This question was recently settled definitively in the negative by Furman [Fur99b] and will be discussed later in this narrative. Adams [Ada88a] earlier had provided another example although it was not an ergodic equivalence relation. One can also show that a measured equivalence relation such that the orbital measures are almost all non-atomic could be generated by the action of a locally compact group - in fact a discrete group cross the circle group. Hence, in a real sense a general measured groupoid as introduced by Mackey does not look a whole lot different from the original examples coming from group actions (except possibly for holonomy groups).

The study of ergodic measurable groupoids actually started out on two different tracksone that we have already described started by Mackey in 1961, and the second one started by Henry Dye in 1959 [Dye59] and [Dye63]. In this remarkable 1959 paper, Dye proved in Mackey's language that all properly ergodic measurable groupoids generated by a free action of the integers and which have finite invariant measure are isomorphic (or equivalently stated, that any such group actions are orbit equivalent). Dye also showed that such an ergodic measured groupoid was hyperfinite or approximately finite in that it was an ascending union of equivalence relations with finite orbits, and conversely that any such relation was generated by a single transformation. Interestingly, Mackey was not aware of the implications of Dye's work for his program of virtual groups until 1972, but Mackey was aware, perhaps by 1971, that the Russian mathematician R. M. Belinskaja had independently proved Dye's orbit equivalence theorem in 1968 [Bel68] [Robert Doran-personal communication]. Belinskaja's mentor V. A. Rokhlin had posed this problem to her so apparently Dye's result was also not known in Russia either. In her proof Belinskaja made essential use of a fundamental result of Vershik [Ver68], who was also a student of Rokhlin. Indeed, both Mackey's papers and Dye's papers were not all that penetrable, and this may have delayed the merging of these two strands for over a decade. The work of Arlan Ramsay [Ram71, Ram76, Ram80, Ram82, Ram85, Ram91, Ram97] and [FeRa85] was influential both in clarifying and simplifying results of Mackey and hence in making them better known, and by a number of significant results concerning the structure of measured groupoids.

One of the first major new developments to flow from these streams was the remarkable work of Wolfgang Krieger extending the work of Dye so as to classify all ergodic actions of the integers up to orbit equivalence [Kr69, Kr70, Kr71, Kr72, Kr76], and which also built on work on von Neumann algebras by Araki and Woods [ArWo69]. All such actions were 
also shown to be hyperfinite so all ergodic integer actions up to orbit equivalence are the same as countable hyperfinite ergodic measured equivalence relations. The classification falls into groups as follows: $I I_{1}, I I_{\infty}, I I I_{\lambda}, 0<\lambda \leq 1$ and $I I I_{0}$, and makes use of the ratio set of the transformation. The ratio set consists of the values that the Radon-Nikodym of the transformation of the measure relative to the measure takes on in some essential way. All but the final class consists of a single isomorphism type, being respectively, transformations preserving a finite invariant measure (Dye), transformations preserving an infinite invariant measure, and transformations where the ratio set is generated by powers of $\lambda$, if $\lambda<1$, or is equal to $[0, \infty)$ if $\lambda=1$. Krieger also shows how to construct for each ergodic countable hyperfinite equivalence relation an ergodic flow of the real numbers, which is determined by the equivalence relation up to conjugacy. He showed that every ergodic flow arises in this way and that the flow up to congugacy is a complete invariant for the ergodic measured equivalence relation with the sole and obvious exception that the flow could not tell the difference between the $I I_{1}$ and the $I I_{\infty}$ case. This flow turns out to nothing but the Mackey range of the Radon-Nikodym cocycle of the hyperfinite measured equivalence relation.

On the other hand, if one starts with a properly ergodic action of the integers, one can construct through the Murray-von Neumann group measure space construction, a von Neumann algebra which is a factor. This algebra depends only on the equivalence relation; that is, it depends on the original transformation only up to orbit equivalence. Hyperfiniteness of the equivalence relation implies hyperfiniteness of the factor. Then Krieger showed that the map from the equivalence relation to the factor was one-to-one so that the hyperfinite factor and the measured ergodic hyperfinite equivalence relation determined each other uniquely. This tied in beautifully with Alain Connes's classification of hyperfinte or injective factors, which had the same classification schema, and Connes [Con73, Con76] showed that all hyperfinite factors with the possible exception of the $I I I_{1}$ case were obtained in this manner from hyperfinite equivalence relations - see also [CoKr77]. The $I I I_{1}$ case remained open until the mid 1980's when it was resolved by Haagerup [Haa87] - see also [Con85a] — who showed that there is only one hyperfinite factor of this kind.

Previously Connes and Takesaki [CoTa77] in their study of the flow of weights of a von Neumann algebra showed that their flow in the case of a group measure factor was the Mackey range of the Radon-Nikodym cocycle, so that the flow of weights for a Krieger factor was the same flow that Krieger had defined. All this closed the loop on a beautiful piece of mathematics which with the exception of the finite measure preserving case, placed in one-toone correspondence hyperfinite factors, countable ergodic hyperfinite measured equivalence relations, and flows of the real line $R$, realized as the flow of weights either of the factor or of the measured equivalence relation. Or if one wants to include the finite measure preserving case, one can rephrase this as a one-to-one correspondence of stable isomorphism classes of hyperfinite factors, stable isomorphism classes of ergodic hyperfinite measured equivalence relations, and flows of $R$ up to conjugacy. In the type $I I$ case the flow is the transitive free flow, In the type $I I I_{\lambda}$ case the flow is the periodic flow for $0<\lambda<1$ with period $\lambda$ (viewed 
as flow of $R^{*+}$ ), is the trivial flow for $\lambda=1$, and is free and properly ergodic for $\lambda=0$.

5. Cartan Subalgebras. Along similar lines, Feldman and Moore [FM77] undertook a study of countable measured equivalence relations observing that when one constructs the von Neumann algebra $M$ from a measured equivalence relation $R$ and a measurable 2-cocycle with values in the (constant field) of the circle group $T$ as an algebra of twisted matrices over the equivalence relation, there is a distinguished abelian subalgebra consisting of diagonal matrices which is isomorphic to $L^{\infty}(X)$ where $X$ is the unit space of $R$. This abelian subalgebra is maximal abelian, has a conditional expectation from $M$ onto it and its normalizer generates the algebra $M$. In analogy with the situation for semi-simple Lie algebras, such algebras of a von Neumann algebra were christened Cartan subalgebras. It was shown that if $A$ is a Cartan subalgebra of a von Neumann algebra $M$ that there is a measured equivalence relation $R$ on a measure space $X$ with $L^{\infty}(X) \cong A$ and a measurable 2 -cocycle with values in $T$ such $M$ is the algebra of twisted matrices over $R$. In the hyperfinite case, the fact that the relation is generated by an action of $Z$ allows one to conclude that any 2-cocycle is trivial so that this added piece of structure does not enter into the picture in this case.

This discussion of course raises the question of whether for any factor von Neumann algebra $R$ there always is a Cartan subalgebra in $R$, and if there are Cartan subalgebras, whether they are unique up to automorphisms of $R$. For hyperfinite algebras, the work described above plus the result of [CFW81] - see section 6 below-demonstrates both of these facts. However it was not long before a counterexample to uniqueness up to automorphisms was found by Connes and Jones [CoJo82] so that two different ergodic measured equivalence yield the same von Neumann algebra. Somewhat later Voiculescu [Vo96], using techniques from free probability theory, found examples of factors which did not have any Cartan subalgebra; indeed he shows that the factor generated by the free group on $n$ generators, $n \geq 2$ does not have a Cartan subalgebra. These outcomes are mildly discouraging but is some sense are not unexpected. On the other side, Sorin Popa in recent work [Popa06a] has shown that if a $I I_{1}$ factor contains a Cartan subalgebra $A$ such that the pair satisfies both a weak rigidity property and a weak amenability property, then any other Cartan subalgebra is conjugate to $A$ by an inner automorphism. This is the first case (aside from the hyperfinite ones) when one knows that invariants of the measured equivalence relation become intrinsic invariants of the von Neumann algebra, a fact that Popa uses to good effect in his article. The notions of amenability and rigidity will be discussed at greater length below.

6. Amenability. Another strand of work emanating from the original papers was to extend Dye's result [Dye59] that applied to actions of the integers to a larger class of groups, and to look at more general measured equivalence relations. Dye himself [Dye63] took the first step in that direction by showing that any abelian group acting in a measure preserving way generates a hyperfinite measured equivalence relation (which therefore can also be generated 
by a action of the integers).

As far as extending consideration to more general groups, a key step was taken by Zimmer when he introduced the notion of an amenable measured groupoid [Zim77, Zim78a, Zim78b] which is cast as a direct generalization of the fixed point property that characterizes amenable groups. He showed that an amenable group acting on a measure space always generates an amenable groupoid, and that the von Neumann algebra generated by an amenable measured groupoid is always hyperfinite. Ornstein and Weiss [OrWe80] proved that any free action of a discrete amenable group with a finite invariant measure generates a hyperfinite equivalence relation. Then Connes, Feldman and Weiss [CFW81] closed the loop and showed that any countable amenable measured equivalence relation is hyperfinite, so that hyperfiniteness and amenability are the same for measured equivalence relations. This was also the key result that showed that two Cartan subalgebras of a hyperfinite factor are conjugate by an automorphism of the algebra - see above. They also showed that any amenable group acting so as to preserve a measure class yields an amenable equivalence relation. In a later paper, Adams, Elliott, and Giordano [AEG94] provide additional information and equivalences. They prove for instance that an a measured groupoid is amenable if and only if the corresponding equivalence relation is amenable and almost all the isotropy subgroups are amenable.

In this context one can obtain one of the first inverse theorems that go from the structure of a measured groupoid or measured equivalence relation to structural result about a group that generates such an groupoid through an action on a space. For this to be successful, it is usually necessary to assume that the action is free (or essentially free - that is free after removing a null set) and that a finite measure is preserved. Under these conditions Zimmer showed that if $G$ acts freely and with a finite invariant measure and if the groupiod or measured equivalence relation generated is amenable, then $G$ must be amenable - see [Zim84]. This works not only for discrete groups, but also for any locally compact group. Thus an amenable equivalence relation does code some information about any group that generates it - namely amenability. This is not much information about the group, but it does provide an glimpse toward much more powerful theorems of this nature to follow. We note that there are easy counterexamples when the the hypotheses fail. For instance if $G$ is a semi-simple Lie group and $P$ is a minimal parabolic subgroup so $P$ is amenable hence the action of $G$ and any subgroup of it, including say a lattice in $G$, on $G / P$ is amenable [Zim84].

7. Trees, Property $(T)$ and Haagerup Property. A concept that bears some relation to amenability is the concept of a countable equivalence relation being treeable. This was introduced by Adams [Ada88b, Ada90] and the condition is simply that there should be a way to assign to each orbit in Borel manner at least on an inessential reduction of the groupoid the structure of a tree - that is a connected acyclic graph. Since a free group on any number of generators has the structure of a tree in an invariant manner, it is evident that the measured 
equivalence relation generated by a free action (or indeed an almost free action) of a free group on a measure space is treeable. Hence any amenable measured equivalence relation is treeable. In the spirit of Mackey's approach Anantharaman-Delaroche has indicated how to extend this to the notion of a countable measured groupoid being treeable [AD05, p. 1010].

A number of interesting and deep properties of treeble equivalence relations were established in the context of Borel category, see [JKL02] which we discuss in section 13. It is evident that these results immediately lead to results in the measured equivalence or measured groupoid context. For instance if follows that treeability for measured equivalence relations is equivalent to being treeable with locally finite trees and that treeability is an invariant of the stable isomorphism class of the relation. The remarkable lifting property established by Hjorth and Kechris [HjKe96, 8.1] comes over and can be extended a bit to apply to measured groupoids as well as measured equivalence relations. So suppose that $G$ is a standard treeable countable measured groupoid and that $H$ is another standard measured groupoid with a surjective map $f$ from $H$ onto $G$ such that it is countable to one. Then there is a lifting - that is a homomorphism of measured groupoids $g$ from $G$ to $H$ such that $f(g(u))=u$ almost everywhere for $u \in G$. This lifting property is very much like the lifting property that characterizes free groups. Hence one might think of treeability of measured groupoids as the analog or extension of the notion of freeness to groupoids. So they could also be called free groupoids.

Just as in the Borel category this result also leads to the following result. Suppose that $E$ countable measured equivalence relation on a space $X$ which is treeable and generated by a group $H$. Then one can construct an action of $H$ on $Y$ such that the action of $H$ is free and such that the measured equivalence relation of $H$ on $Y$ is stably isomorphic to the original equivalence relation $H$ generates on $X$. This is one answer to the question left open in [FM77] about groups acting freely, but this result also raises the question of whether any countable measured equivalence relation is stably isomorphic to one that is generated by a free action of a countable group. Furman's examples that we will discuss shortly also provide counterexamples to this question as well as to the original question in [FM77].

In 1967 Kazhdan [Kaz67] introduced the seminal notion of group having Property $(T)$, which briefly stated means that the one-dimensional trivial unitary representation is an isolated point in the unitary dual space of $G$ with respect to the Fell topology. This has proved of great importance for both discrete groups as well as continuous groups for if $H$ is a closed subgroup of $G$ such that $G / H$ has a finite invariant measure, then if $G$ has Property $(T), H$ does too. Property $(T)$ is the very opposite of amenability in that an amenable group which also has Property $(T)$ is necessarily compact. Also it is obvious that free groups never have Property $(T)$. Zimmer [Zim81] showed how to define a corresponding Property $(T)$ for actions of a countable group $G$ on a measure space $X$, which is really a property of the measured groupoid $X \times G$, and proved a number of results. Anantharaman-Delaroche [AD05] extended the definition to a general countable measured groupoid. Property $(T)$ is equivalent to the vanishing of certain one-dimensional cohomology groups [AD05, §4.3]. 
Standard results are that if $G$ has Property $(T)$ and acts to preserve a finite invariant measure, then the groupoid $X \times G$ has Property $(T)$ and conversely if $X \times G$ has Property $(T)$ then $G$ has Property $(T)$. A transitive action of $G$ on $H / G$ has Property $(T)$ if and only if $H$ has Property $(T)$ [AD05] so that the property for groupoids is a generalization of the property for groups in the spirit of Mackey's notion of virtual groups. Property $(T)$ for countable measured groupoids is an invariant under stable isomorphism [AD05], and if a measured groupoid has Property $(T)$, then the corresponding measured equivalence relation also has Property $(T)$. Finally if $G$ is properly ergodic and has Property $(T)$, then it cannot be amenable, and indeed it cannot be treeable either, [AdSp90] and [AD05]. Connes and Jones have also formulated a Property $(T)$ for von Neumann algebras-[CoJo85], but we will not explore that notion here.

Another very interesting property that a locally compact group may or may not have, which is related to amenability and Property $(T)$, is the Haagerup Property. This appeared in various different guises, perhaps first in Haagerup [Haa79], but more explicitly in [AkWa81] and [Ch83], but then in a very different guise in Gromov's program in geometric group theory [Gro88] and [Gro93] where he named it a- $T$-menability as kind of "cross" between amenability and Property $(T)$. All the different definitions are known to be equivalent - see [CCJJV01]. The formulation that Mackey would have liked best is that there exists a unitary representation, all of whose matrix coefficients tend to zero at infinity, but which weakly contains the trivial one-dimensional representation. This demonstrates immediately that amenability implies the Haagerup property as a characterization of amenability is that the regular representation, whose coefficients do tend to zero at infinity, weakly contains the trivial one-dimensional representation. It also demonstrates that a group can have the Haagerup property and Property $(T)$ only if the group is compact. Hence the Haagerup class contains all amenable groups, but is strictly larger as free groups and free products of amenable groups have the Haagerup property as do the rank one simple Lie groups $S O(n, 1)$ and $S U(n, 1)$. The connected Lie groups with Haagerup property can be completely classified-[CCJJV01, p. 41]. There is a related property called $K$-amenability introduced by Cuntz [Cun83], which is now known to be implied by the Haagerup Property [HiKa97], [HiKa01], and also [Tu99b] and [Tu00]. There are groups that are $K$-amenable but do not have the Haagerup property, for example the semi-direct product of $Z^{2}$ with $S L_{2}(Z)$ - [CCJJV01, p. 8].

In the spirit of Mackey's idea of virtual groups, it would be natural to seek to make sense of the Haagerup property for measured equivalence relation or measured groupoids. Jolissaint [Jo05] has done exactly this at least for measure preserving relations. In fact a little earlier $\mathrm{Tu}[\mathrm{Tu} 00]$ had defined exactly such a property for topological groupoids (also see below in section 10). One could perhaps pick nits and argue that in the title of Tu's paper one should substitute "a-T-moyennables" for "moyennables". A corresponding definition of the Haagerup for measured groupoids is not currently in the literature, but would be easy to formulate. In any case in the measure preserving case, Jolissaint shows that the Haagerup property for measured equivalence relations is a stable isomorphism invariant, and that if 
discrete group acts freely on measure space $X$ and generates a measured equivalence $R$ with the Haagerup property, then $G$ has the Haagerup property. He notes that the hoped for converse of this that if $G$ has the Haagerup property so does $R$ is not known. It is an interesting question whether a treeable measured equivalence relation, which from the discussion above would seem to be the groupoid analog of freeness for a group, must have the Haagerup property.

8. Rigidity. We continue with the theme of examining properties of measured groupoids or measured equivalence relations which code properties of a group that acts on a measure space $X$ so as to generate the groupoid or equivalence relation. We have already seen some examples of this phenomenon for amenability, Property $(T)$, and the Haagerup Property. Henceforth we shall consider just ergodic countable measured equivalence relations which have a finite invariant measure and free actions of discrete groups. First of all there is a significant invariant of an ergodic measured equivalence equivalence relation $R$ with a finite invariant probability measure $\mu$ called the fundamental group of $R$. For any $t, 0<t<1$, one can form a new equivalence relation $R_{t}$, by choosing a subset $Y$ of the unit space $X$ of $R$ of measure $t$, then restricting the relation to $E$, forming $\left.R\right|_{Y}$, and then re-scaling the restriction of the probability measure on $Y$ by $1 / t$, so that it becomes a probability measure on $Y$. By ergodicity, this construction is up to isomorphism independent of the choice of $Y$ and depends only on $t$. One can similarly construct $R_{t}$ for $t>1$ so that $\left(R_{s}\right)_{t}$ is isomorphic to $R_{s t}$. Of course, all the $R_{t}$ are stably isomorphic to each other, but they may not be isomorphic. The fundamental group of $R$ is the subgroup of $R^{*+}$ of all $t$ so that $R_{t}$ is isomorphic to $R$. It should also be remarked that the $R_{t}$ are exactly the countable ergodic measured equivalence relations with an invariant probability measure that are stably isomorphic to $R$.

This notion is clearly based on the prior notion of the fundamental group of a type $I I_{1}$ factor $M$, which consists of all $t$ in $R^{*+}$ with $M_{t}$ (suitably defined) isomorphic to $M$. If $M$ is the type $I I_{1}$ generated by an equivalence relation $R$ via the group measure construction, the fundamental group of $R$ is clearly a subgroup of the fundamental group of $M$ as an isomorphism of $R$ with $R_{t}$ induces an isomorphism of $M$ with $M_{t}$. If the corresponding Cartan subalgebra $A$ of $M$ is unique up to automorphisms, then the two fundamental groups agree as any isomorphism of $M$ with $M_{t}$ leads to an isomorphism of $R$ with $R_{t}$. This is the basis for the argument of Popa in [Popa06c] to produce an example of a $I I_{1}$ factor with trivial fundamental group from a equivalence relation with trivial fundamental group for which the Cartan subalgebra is unique up to automorphisms. Of course the fundamental group of hyperfinite type $I I_{1}$ equivalence relation is all of $R^{*+}$ by Dye's theorem, and it has long been known that the fundamental group of the type $I I_{1}$ factor is all of $R^{*+}$. Constructing equivalence relations and factors with small or trivial fundamental groups is more challenging; see [Con80] and [GeGo88]. Popa [Popa06c, I, Corollary 0.4] has shown how, given any countable subgroup $S$ of $R^{*+}$, to construct an ergodic $I I_{1}$ countable ergodic measured relation $R$ whose fundamental group is exactly $S$. 
Zimmer in pioneering work that built on Margulis's rigidity theorems, established striking and deep rigidity theorems for actions of lattices in simple groups of higher rank. Subsequently Furman made some improvements in these results so that now one has quite definitive results - [Zim80, Zim81, Zim84], [Fur99a, Fur99b]. The setup is that $\Gamma$ is a lattice in a simple Lie group $G$ of real rank greater than or equal to two with finite center, and that $\Gamma$ acts essentially freely (free except on a null set) and ergodically on a space $X$ leaving a finite invariant measure invariant. Before describing the results we need a few preliminaries. Examples of such $\Gamma$ actions are the natural actions of $\Gamma$ on $G / \Delta$ for some other lattice $\Delta$ in $G$. It is important to note that the action of $\Gamma$ on $G / \Delta$ and the action of $\Delta$ on $\Gamma \backslash G$ are stably orbit equivalent. As a generalization of this construction, consider a $\Gamma$ action on $X$ and suppose that there is an equivariant quotient map $p$ of $X$ to the $\Gamma$ space $G / \Delta$ for some other lattice $\Delta$ in $G$. Then one can define a space $X_{p}$ with an essentially free $\Delta$ action on $X_{p}$ such that the measured equivalence relation on $X_{p}$ generated by $\Delta$ is stably orbit equivalent to the measured equivalence relation generated by $\Gamma$ on $X$ [Fur99b].

The Zimmer-Furman results are as follows: we have $\Gamma$ a lattice in $G$ as above, and suppose that $\Lambda$ is another countable group that acts essentially freely on a space $Y$, and that the measured equivalence relations so generated on $X$ by $\Gamma$ and $Y$ by $\Lambda$ are stably isomorphic. Then first of all $\Lambda$ is up to finite groups isomorphic to a lattice $\Delta$ in the same Lie group $G$ where $\Gamma$ sits. But then moreover, if the $\Gamma$ space $X$ has no quotients of the form $G / \Delta$, then up to finite groups $\Delta$ is isomorphic to $\Gamma$, and the actions on $X$ and $Y$ are isomorphic up to finite groups. If on the other hand, this is not the case, then there exists a $\Delta$ such that the $\Gamma$ space $X$ does have an equivariant map $f$ onto $G / \Delta$ for some lattice $\Delta$ in $G$, such that the action of $\Lambda$ on $Y$ is isomorphic up to finite groups to the $\Delta$ action on $X_{f}$ as constructed above. Thus, the stable isomorphism class of the equivalence relation $R$ generated by $\Gamma$ on $X$ codes a lot of information: first of all, it codes the Lie algebra of the ambient simple Lie group $G$ and also it restricts the actual isomorphism type up to finite groups of the action itself to a discrete and understandable set of examples. So we can go from stable orbit equivalence to isomorphism of actions, indicating that the measured equivalence relation is indeed very rigid.

Furman goes on to use his techniques to resolve the issue of whether for an ergodic countable measured equivalence relation $R$ on $X$, one can find a countable group acting essentially freely on $X$ which generates $R$. Indeed he shows that if $R$ is one of the measured equivalence relations generated by a free action of a lattice $\Gamma$ in a higher rank simple group, then $R_{t}$ (as defined three paragraphs above) for most values of $t$ (all but a countable set) will never be generated by a free action of any group [Fur99b, Theorem D]. Of course these examples are stably isomorphic to a relation that is generated by a free action, but using a different kind of example, Furman can produce examples of ergodic measures equivalence relations that are not stably isomorphic to anything that is generated by a free action [Fur99b, Theorem D and Remark 1.4].

Gromov in his work on the geometric theory of groups has introduced the notion of two 
groups being measure equivalent [Gro93] and see [Sh05]. Briefly two countable groups $\Gamma$ and $\Delta$ are measure equivalent if they have commuting measure preserving free actions on an infinite Lebesgue space each with a fundamental domain of finite measure. Furman in [Fur99a] shows that this is the same as the two groups having finite measure preserving free ergodic actions such that the measured equivalence are stably isomorphic. In this language, the Zimmer-Furman results say that the measure equivalence class of a lattice in a higher rank simple Lie group $G$ consists of those groups which are, up to finite groups, lattices in the same Lie group $G$. Also discrete amenable groups form a single measure equivalence class.

Finally let us note that Monod and Shalom, by using techniques from the theory of bounded cohomology of groups developed by Burger and Monod [BuMo02] and [Mon01] have obtained a wealth of new rigidity results especially concerning products of groups, which are contained in [MoSh04], [MoSh06] and [Mon06]. They use these results to address the questions about how many non-isomorphic measured equivalence relations can be generated free actions of a given group. Recall that an amenable group can only generate one such isomorphism type. Monod and Shalom [MoSh06, Theorem 1.7] show among many other things that there are power of the continuum different finitely generated torsion free groups each of which has uncountable many free ergodic measure preserving actions such that no two measured equivalence relations in this family are stably isomorphic. It should be observed that Gaboriau and Popa [GaPo05] have shown that any free group on at least two generators also has uncountably many free ergodic measure preserving action whose measured equivalence relations are not stably isomorphic. Finally Hjorth [Hj05a] has shown for any infinite discrete group with Property $(T)$, there are uncountably many free ergodic actions that are not orbit equivalent, extending earlier work in [GeGo88] for arithmetic groups with Property $(T)$.

9. Betti Numbers and Cost. Another instance of equivalence relations coding properties of groups arises from the $L_{2}$ Betti numbers of a discrete group as defined by Cheeger and Gromov [ChGr86]. Cheeger and Gromov through introduction of a singular $L^{2}$ cohomology are able to define Betti numbers for any discrete group $G, \beta_{n}(G)$. Gaboriau [Gab02] has shown how to define a simplicial structure on a measured equivalence relation $R$ and assuming always that there is a finite invariant measure, he can define $L^{2}$ cohomology groups and then Betti numbers $\beta_{n}(R)$ of the ergodic measured equivalence relation. These are first cousins of the $L^{2}$ Betti numbers of a measured foliation as defined by Connes in [Con79]. The point here is that if $G$ acts freely or essentially freely preserving a finite invariant measure to generate the measured equivalence $R$, the $\beta_{n}(G)=\beta_{n}(R)$. Hence the equivalence relation $R$ encodes the Betti numbers of any group that acts freely to generate it. The Betti numbers are clearly isomorphism invariants of the measured equivalence relation, and they scale under stable isomorphism. Recall that if $R$ is a countable measured ergodic equivalence relation with an invariant probability measure, then the countable measured equivalence relations 
with invariant probability measures that are stably isomorphic to $R$ are exactly the $R_{t}$. Then for all $t>0, \beta_{n}\left(R_{t}\right)=\beta_{n}(R) / t$ for all $n$. As a corollary, it follows that if any $\beta_{n}(R)$ is not equal to zero or infinity, its fundamental group must be trivial.

Since any amenable (hyperfinite) equivalence relation $R$ is generated by an action of the integers $Z$, and as the Betti numbers of $Z$ are all zero, the Betti numbers of $R$ are all zero. These results can also be used to distinguish equivalence relations generated by actions of lattices in semi-simple Lie groups, as the Betti numbers of such a lattice vanish except possibly when $n$ is the dimension of the corresponding symmetric space [Gab02, 1.6]. One may conclude for instance that equivalence relations generated by a lattice in $S p(n, 1)$ cannot be stably isomorphic to one generated by $S p(m, 1)$ only if $m=n$, and similarly for $S U(n, 1)$ and $S U(m, 1)$ and for $S O(2 n, 1)$ and $S O(2 m, 1)$ [Gab02, Corollary 0.4]. These results for rank one groups complement the much stronger results that we have already described for lattices in higher rank groups. A relation generated by a free action of a free group on $\mathrm{n}$ generators has first Betti number equal to $n-1$ with all others zero, so relations generated by free actions of free groups on $n$ and $m$ generators can be stably isomorphic only if $m=n$ $[$ Gab02, 1.6].

Another invariant of a measured ergodic equivalence relations with a finite invariant measure is the cost of the relation $R$ on a space $X$ that was first introduced by Levitt [Lev95], and then greatly expanded upon by Gaboriau [Gab00]. The idea here is that one looks at a generating system - that is, a set of partially defined maps $f_{j}: A_{j} \rightarrow B_{j}$ which are isomorphisms of the relativized relations on $A_{j}$ and $B_{j}$ and such that $R$ is the smallest relation such that $x \sim f_{j}(x)$ for all $x \in A_{j}$ and all $j$. Such a system is called a graphing of $R$. The cost of the graphing is the sum of the measures of the $A_{j}$, and the cost of the relation $R$, written $c(R)$ is the infimum of the costs of all possible graphings. A graphing induces the structure of graph on each or at least almost all orbits, and if this graph turns out to be tree for almost all orbits, the relation is treeable as defined above, and the graphing then is said to be a treeing. It is an important result that the cost of a treeing of a relation relation $R$ achieves the minimum among the costs of all graphings and is hence equal to the cost of the relation [Gab00, Theorem IV.1], and conversely if the cost of the relation is finite and the minimum is achieved, the graphing is a treeing [Gab00, Proposition I.1]. Hjorth in [Hj06] has obtained an interesting result about how this minimum can be obtained.

Assuming that $R$ is properly ergodic for convenience, there is a simple scaling law for the cost of relations that are stably isomorphic; namely $\left(c\left(R_{t}\right)-1\right)=(c(R)-1) / t$ for any $t>0$. Hence having cost 1 (or infinity) is a stable isomorphism invariant for $R$ [Gab00, Proposition II.6]. It is evident that a free action of a free group on $n$ generators has cost exactly $n$ because there is a natural treeing. Gaboriau then defines the cost of countable group $G$ to be the infimum of the costs of the free actions of $G$ and that a group is said to have fixed cost if all a free actions have the same cost. He shows that for any countable group, there is always free action that that achieves the cost of the group [Gab00, Proposition VI.21]. It is evident that the cost of a free group on $n$ generators, $F_{n}$ is $n$ and that $F_{n}$ has fixed cost. 
Many interesting groups have cost 1 including all lattices in a semi-simple Lie group of rank greater than one [Gab00, Corollary VI.30].

10. Topological Groupoids. Another direction in which Mackey's ideas of a virtual group have had an impact is in the study of topological groupoids. With the introduction of topology and geometry into the groupoid structure, we move into the realm of what is called non-commutative geometry, whereas what we have been talking about for the last several sections with measured groupoids could be described as non-commutative functional analysis. Just as measured groupoids have an intimate connection to von Neumann algebras, topological groupoids will have the same kind of connection with $C^{*}$-algebras.

We will restrict here to locally compact topological groupoids $G$ - that is groupoids $G$ where $G$ and the unit space $X$ are locally compact (and as for groups are also second countable), and multiplication, range, source, and inverse maps are continuous. We will also assume that the range and source maps are also open maps. For simplicity we will assume for the moment that $G$ is Hausdorff although we realize that the theory has to be developed for groupoids that are locally Hausdorff so that one of the principal examples - the groupoid of a foliation or a foliated space, which may be only locally Hausdorff are not excluded. Of course topological groupoids appear whenever a locally compact group acts as a topological transformation group on a locally compact space, although the groupoid point of view did not come to the fore until later in the development of the topic. Early on it was clear that groupoids were key in studying foliations and that there was a natural holonomy groupoid associated to any foliation, although the details of this were not written down until somewhat later--[Wi83]. The groupoid and operator algebra connection with foliations emerged in Connes's work [Con79] and [Con82]. Renault [Ren80] laid out systematically the connection between topological groupoids and $C^{*}$-algebras. Thus it was only in the late 1970's some fifteen years after Mackey's first writings on groupoids and virtual groups that these ideas about topological groupoids and non-commutative geometry jelled and the field began to thrive.

In [Ren80], the author lays out definitions and specifically the definition of a continuous Haar system for a locally compact groupoid $G$, and then the construction of the $C^{*}$-algebra of the groupoid or more properly the reduced $C^{*}$-algebra $C_{r}^{*}(G)$ of the groupoid obtained by completing a convolution algebra of function on $G$ in the norm from the regular representation. The full groupoid $C^{*}$-algebra comes by completing this convolution algebra under a larger norm coming from other representations of the groupoid. There is always a surjection of this latter $C^{*}$-algebra onto the reduced $C^{*}$-algebra. This construction just as in the von Neumann algebra case allows for the presence of a 2-cocycle with values in the circle group which now has to be continuous. This construction is not really new because just as the group measure construction of von Neumann algebras goes back to the 1930's, the $C^{*}$-algebra constructed from the action of a locally compact group on a locally compact space goes back many years and has a venerable history. What is new is the insight and 
flexibility that the groupoid point of view yields.

Because a (locally) compact space $X$ is completely characterized by $C(X)\left(C_{0}(X)\right)$, the two are in some sense interchangeable. But when we come to a "topological" space such as the quotient of a manifold by the leaves of a foliation, or the quotient of compact space by an action of group that may dense orbits, or more generally the quotient of the unit space of a groupoid by the orbits of the groupoid, this space, while of geometric and topological interest, may have no continuous functions at all. As Connes has argued, we should regard the $C^{*}$ algebra of the groupoid as the replacement for $C(X)$ in this non-commutative situation. This $C^{*}$-algebra does carry important geometric and topological information and its structure is at the heart of non-commutative geometry and topology.

One obvious point of interest about a $C^{*}$-algebra substituting as it were for a commutative algebra and its underlying topological space is the space of primitive ideals of this noncommutative algebra. This question arose very early on in the work of Effros and Hahn [EfHa67] on the structure of transformation group $C^{*}$-algebras. They posed a conjecture about primitive ideals which was subsequently called the Effros-Hahn conjecture that was resolved by Gootman and Rosenberg [GoRo79]. This result was subsequently extended to the groupoid case by Renault [Ren91] so that one has this result available for groupoids.

Of course the $C^{*}$-algebra of a group provided many significant examples of $C^{*}$-algebras, as did the $C^{*}$-algebras of topological actions of a locally compact group on topological spacethe group action $C^{*}$-algebra. Beginning with [Ren80] if became clear that a number of other significant examples of $C^{*}$-algebras that did not at first appear to have any connections with groupoids could be brought under the $C^{*}$-groupoid construction. This included the $A F$ algebras as well as the Cuntz and Cuntz-Krieger algebras [Cun83], [CuKr80] as well as others - see also [AD97], [KPRR97], [KPR98], [Ren00] and [ExRe06]. The $C^{*}$-algebras of foliations, foliated spaces, and laminations also obviously fall under this rubric. While it cannot be claimed as in the von Neumann algebra case that essentially all interesting von Neumann algebras arise from a groupoid construction, it is a plausible claim that for $C^{*}$ algebras, essentially all interesting ones are extensions of a groupoid $C^{*}$-algebra by another groupoid $C^{*}$-algebra.

There is also a natural concept of a discrete topological groupoid, analogous to the notion of countability in the measured groupoid case, where requires that the range and source maps from $G$ to the unit space $X$ be local homeomorphisms. By second countability, the range and source maps are countable to one, and as is observed in [Ren80] this hypothesis ensures the existence of a continuous Haar system. We remark that discrete groupoids are also called étale groupoids.

The notion of equivalence or similarity for topological groupoids is somewhat more subtle than the corresponding notion of similarity or stable isomorphism for measured groupoids where this notion amounted just to isomorphism after "fattening up" the orbits. The paper [Ren82], see also [MRW87], provides an effective definition of equivalence of locally compact groupoids $G$ and $H$ which is that here is a locally compact space $Z$ which is both a principal 
$G$ and principal $H$ space with the actions commuting and so that the unit space $X$ of $G$ is homeomorphic to the quotient $Z / H$ and the unit space $Y$ of $H$ is homeomorphic to $G \backslash Z$. This definition has much in common with Gromov's definition of measure equivalence discussed earlier.

However it is possible to rephrase this definition of equivalence, which is often called Morita equivalence of topological groupoids, in a way that more resembles the definition in section 3 above of equivalence, or stable isomorphism for measured groupoids. Recall that stable isomorphism for measured groupoids means isomorphism after "fattening up" the orbits by crossing them with the unit interval with Lebesgue measure. Now, suppose that $G$ is a topological groupoid with unit space $X$. Suppose further that $Y$ is another topological space and that $p$ is an open continuous surjection of $Y$ onto $X$. Following [Tu04] we can "blow up" $G$ along the map $p$ by forming the fibered product of $G$ and $Y \times Y$ over $X \times X$ by means of $(r, s)$ and $p \times p$ to form a new topological groupoid denoted $G[Y]$. This new groupoid has $Y$ as its space of units and and the orbits of the groupoid $G[Y]$ in $Y$ are simply the inverse images of the orbits of $G$ in $X$. Hence one can think of this process as "fattening up" the orbits of $G$ by replacing them with their inverse images under $p$ in $Y$. Everything else remains the same, that is, $G$ and $G[Y]$ have the same orbit space and the same holonomy groups. Then by Proposition 2.29 of [Tu04], two topological groupoids $G$ and $H$ with unit spaces $X$ and $Y$ respectively are Morita equivalent if and only if there is a topological space $Z$ with continuous open surjections $p$ and $q$ onto $X$ and $Y$ respectively such that $G[Z]$ and $H[Z]$ are isomorphic as topological groupoids. Hence Morita equivalence can be interpreted as isomorphism after "fattening up" the orbits.

If $G$ is an étale groupoid, and $Y$ and $p$ are as above, then $G[Y]$ need not be an étale groupoid. However if $p$ is an étale map - that is a local homeomorphism from $Y$ onto $X$, then it is clear that $G[Y]$ is indeed an étale groupoid. Then the natural definition of Morita equivalence for étale groupoids $G$ and $H$ with unit spaces $X$ and $Y$ respectively, cf. [Hae01], is that there is a space $Z$ with étale mappings $p$ and $q$ onto $X$ and $Y$ respectively such that the étale groupoids $G[Z]$ and $H[Z]$ are isomorphic. We note that this definition of Morita equivalence for étale groupoids parallels very closely the definition of stable isomorphism for discrete measured groupoids in section 4 where two such groupoids are equivalent or stably isomorphic if they become isomorphic after "fattening up" all orbits by crossing them with a copy of the integers $Z$.

In any case the authors in [MRW87] show that the full $C^{*}$-algebras are strongly Morita equivalent and hence with our conditions of second countability, they are stably isomorphicthat is $C^{*}(G) \otimes K \cong C^{*}(H) \otimes K$ where $K$ is th algebra of compact operators on separable Hilbert space. This applies for instance to the case of the $C^{*}$-algebra of foliation and the $C^{*}$-algebra of a complete transversal. It is also true that the same result on strong Morita equivalence hold for the reduced $C^{*}$-algebras of equivalent groupoids. The authors of [MRW87] have told the author that this result should have been included in the original paper and that if follows by the same kind of reasoning. 
For measured groupoids, and also as we shall see for Lie groupoids below, there is simple criterion for one of these kinds of groupoids to be equivalent or similar to a discrete or étale groupoid. In both of these categories the simple condition is that all (or almost all) the holonomy groups should be discrete. A corresponding result appears to be unknown for locally compact groupoids.

Recall from section 3 that one can define a groupoid cohomology theory for a measured groupoid $G$ with coefficients in a measurable field of Polish abelian groups $A$ on which $G$ operates as module - see [Ser81]. This poses the question of whether one could define a suitable cohomology theory for locally compact groupoids with coefficients in a continuous field of abelian Polish groups on which $G$ operates continuously. Such a cohomology theory would be more an analytic object with connections to $C^{*}$-algebras (the Dixmier-Douady classification theory), unitary representations, and groupoid extensions, but also with connections to the topology of the underlying structure. Such a theory should reduce in the case when the unit space is a point to the group cohomology of a locally compact group with coefficients in a Polish module (to the theory described in [Moore76]). When the groupoid is equal to its unit space, such cohomology should reduce to sheaf cohomology of the unit space. One also has a pretty good idea of what the low dimensional groups should look like and in particular, $H^{2}(G, T)$ should be the Brauer group of the locally compact groupoid as defined and studied in [KMRW98]. Some clues might come from [Hae79], [Hae01], [Moer95], [Moer01] or [Kum88] for the étale case, but the theory defined by $\mathrm{Tu}$ in [Tu06] seems to fit the bill very nicely and has all the good properties, including invariance under Morita equivalence.

When the groupoid is discrete, the unit space $X$ is an open subset of $G$ and it is easy to see that the $C^{*}$-algebra of continuous functions vanishing at infinity, $C_{0}(X)$, is a subalgebra of the any twisted reduced $C^{*}$-algebra of the groupoid $G$. The twist come about by a continuous two-dimensional cocycle with coefficients in the circle group. If the groupoid is principal then this subalgebra enjoys properties as a subalgebra of the $C^{*}$-algebra of the groupoid that are quite analogous to those that Cartan subalgebra enjoys as a subalgebra of a von Neumann algebra (maximal abelian, with conditional expectation and normalizer generating the big algebra) and Renault in [Ren80] calls such a subalgebra a Cartan subalgebra and under appropriate conditions show that just as in the von Neumann case there is a converse that says that any $C^{*}$-algebra with a Cartan subalgebra arises from this construction with possibly a cocycle. Subsequently Kumjian in [Kum88] expanded and clarified these result, introducing a kind of subalgebra that he calls a diagonal and obtains a converse result that such a diagonal comes from a discrete equivalence relation and what he calls a twist. Thus the theory to a point parallels the Cartan subalgebras of measured groupoid von Neumann algebras.

As a further parallel to the measured groupoid and group situation, Renault introduced in [Ren80], the notion of an amenable topological groupoid and established a number of results. In subsequent work joint with Anantharaman-Delaroche [ADRe00] the authors in very elegant manner expand and clarify the notion of amenability for topological groupoid 
and compare and contrast the definition with the definition in the measured groupoid case (section 6 above), and in the case of Borel equivalence relations (see section 12 below). They show that topological amenability is invariant under equivalence of topological groupoids in the sense of [MRW87]. Moreover, if $G$ is a topological groupoid with a Haar system and if $G$ is topologically amenable, it is measurewise amenable in the sense that $G$ is amenable as a measured groupoid for all quasi-invariant measures with respect to the given Haar system. The converse is true if $G$ is topologically equivalent to a groupoid with a continuous Haar system and with countable orbits [ADRe00, pp. 84-86], and hence this is true for any étale groupoid or anything topologically equivalent to an étale groupoid.

It is also true, as expected based on the analogy with groups, that topological amenability implies that the full group $C^{*}$-algebra is equal to the reduced $C^{*}$-algebra. Indeed if $G$ is a topological groupoid with a continuous Haar system, and is measurewise amenablehence in particular if it is topologically amenable - then $C^{*}(G)=C_{r}^{*}(G)$. Likewise in the same context, measurewise amenability for $G$ implies that $C^{*}(G)$ is a nuclear $C^{*}$-algebra. Conversely if all isotropy groups of $G$ are discrete, then nuclearity of $C_{r}^{*}(G)$ implies that $G$ is measurewise amenable [ADRe00, pp. 146-153]. We should add that this little gem of a book also contains many very interesting additional results that for lack of space we cannot include here. Again, following the lead from group theory, one can introduce the notion of a topological groupoid having the Haagerup property or being a- $T$-menable. The definition is implicit in [Tu99a, Tu99b] and more explicit in [Tu00] in which certain properties of the $C^{*}$-algebra of the groupoid are established that are parallel to properties of the $C^{*}$-algebra of an group with the Haagerup property.

11. Non-commutative Algebraic Topology. Given that $C^{*}$-algebras of groupoids or $C^{*}$-algebras generally are prime objects of study in non-commutative topology and geometry, one needs for their study the analogs of the usual tools of algebraic topology - namely (co)homology and $K$-theory. Of these $K$-theory is the most transparent for it was realized early on that the $K$-theory of a compact or locally compact space topological space as initially defined by vector bundles had a very simple interpretation as the $K$-theory of the corresponding $C^{*}$-algebra of continuous functions (vanishing at infinity) on the space. In some ways the interpretation in terms of the $C^{*}$-algebra was even more natural than the classical vector bundle approach. When it came to $K$-homology of a space, it became clear that the only effective way to proceed was via $C^{*}$-algebras as illustrated by [BDF77] for the odd dimensional case and by Atiyah [Ati76] and Kasparov [Ka75] in the even dimensional case; see also [Dou80] and [BaDo82]. Then Kasparov discovered the very elegant and powerful bi-variate approach with his $K K$-theory [Ka80] where $K$-theory groups $K K(A, B)$ are defined for a pair of $C^{*}$-algebras $A$ and $B$. These groups include regular $K$ groups for $K(A, \mathbb{C})$ is $K$-homology of $A$ while $K K(\mathbb{C}, B)$ is $K$-cohomology of where $\mathbb{C}$ is the complex numbers. The $K K$ groups come equipped with a Kasparov product, which is powerful and essential tool for their use. If $A$ and $B$ are commutative one ends up with new insights 
even in the classical case. One also has Connes's analog of the Thom isomorphism theorem [Con81], which calculates the $K$ groups of the crossed product $C^{*}$-algebra $A \ltimes R$ of $A$ by an action of the real line on $A$ in terms of the $K$-theory of $A$. This is an essential tool for calculating $K$ groups of $C^{*}$-algebra that arise from groupoid constructions.

One extraordinarily important use of the $K$ groups and $K$-theory is in generalizations of the Atiyah index theorems for families. As developed by Atiyah and Singer, one has a family of elliptic operators along the fibers of a fibered space with the fibers indexed by a base space $B$. The index of the family ends up being an element of the $K$-theory of the base space, and the theorem for families calculates this index in terms of topological invariants, or to put it another way it equates the analytic index of the family of operator to a topological index and it gives a formula for it in terms of topological invariants. If one now considers a foliation or a foliated space with elliptic operators on each leaf of the foliation, one has again a family of elliptic operators but which is now indexed by the non-commutative space of the set of leaves of the groupoid $G$ of the foliated space. This is exactly the situation that the $C^{*}$-algebra of the foliated space is designed to handle, and the index of the family becomes an element of of the $K$-theory of $C_{r}^{*}(G)$. The index theorem equates this analytical index to a topological index built out of topological invariants as described in [Con79], [Con82] and [CoSk84]; see also [MoSc06].

A another key tool in understanding and working with the $K$-theory of groupoid $C^{*}$ algebras is the Baum-Connes conjecture which dates from about 1980, even though their original paper [BC00] was not published until 2000; but see also [BC88a], [BC88b] and [BCH94]. The conjecture was originally stated for discrete groups but its domain was extended to include continuous groups, foliation groupoids, and then locally compact groupoids [Tu00]. So let $G$ be a locally compact Hausdorff groupoid with a continuous Haar system and $C_{r}^{*}(G)$ be its reduced $C^{*}$-algebra. Then the idea is to associate to $G$ a topological space $E G$ as kind of classifying space for proper actions of $G$. Then there is an analytic assembly map from the $K$-homology of $E G$, suitably defined inductively, to the $K$-theory of the reduced $C^{*}$-algebra of the groupoid $G$. This map is conjecturally an isomorphism-the Baum-Connes conjecture. The point here is that $E G$ is in some sense a fairly standard geometric object where one can make computations, and hence the structure of the $K$-theory in the highly non-abelian case of $C_{r}^{*}(G)$ can be reduced to these computation in the commutative case.

The conjecture has been established in a wide variety of special cases in the years since it was first proposed. For instance Higson and Kasparov [HiKa97], [HiKa01] showed the Baum-Connes Conjecture for discrete groups with the Haagerup property (a- $T$-menable). $\mathrm{Tu}[\mathrm{Tu} 00]$ extended this to show that the Baum-Connes conjecture holds for groupoids with the Haagerup property. However Property $(T)$ groups had resisted analysis for many years until Lafforgue's [Laf02] breakthrough in which he established the result for some Property $(T)$ groups as a consequence of much more general results. Using these results, Mineyev and $\mathrm{Yu}$ [MiYu02] showed that the conjecture holds for all subgroups of Gromov hyperbolic groups, thus extending an earlier result of Connes and Moscovici [CoMo90] who showed 
that the assembly map in injective for hyperbolic groups. Tu has defined the notion of a hyperbolic foliation and showed that the assembly map is injective for the groupoid of such a foliation. Thus positive results on the Baum-Connes conjecture for groups are being extended to groupoids. However recently counterexamples to the conjecture for groupoids, but not for groups, have been discovered-[HLS02] so it is not clear what the general situation will be.

The validity of the conjecture in cases where it can be established has many important and significant consequences, including the Novikov Conjecture on homotopy invariance of higher signatures of manifold with fundamental group equal to the discrete group $G$; in fact rational injectivity of the assembly map will imply this as noted by Mishchenko [Misc74]. The validity of the Gromov-Lawson-Rosenberg Conjecture [Ros83, Ros86] also follows from the Baum-Connes conjecture.

One can get a better understanding of the construction of the assembly map and the conjecture by Iooking at one of the simplest special cases when $G$ is a discrete torsion free group that is the fundamental group of a compact manifold $M$ with contractible universal covering. In this case $E G$ is just $M$, and in fact consider the special case of this when $G$ is abelian, hence is $Z^{n}$, and $M$ is a torus $T^{n}$. Lusztig [Lus72] show how to take an elliptic operator on a vector bundle over $M$, which one also views as representing an element of the $K$-homology of $M$, and then tensor the bundle with a character $\chi$ of $G$, which is realized as the fundamental group of $M$ and obtain a twisted version of the elliptic operator on the twisted bundle. The result is a family of elliptic operators indexed by the dual group $\hat{G}$. Then by the Atiyah-Singer index theorem for families, the index of this family is an element of the $K$-theory of the $C^{*}$-algebra $C(\hat{G}), K(C(\hat{G}))$. But by Fourier transform $C(\hat{G})$ is simply the $C^{*}$-group algebra of $G$ so we get a way to map $K$-homology of $M$ into the $K$-theory of $C^{*}(G)$ as desired. If now $G$ is no longer abelian, the dual group is no longer there to facilitate this simple construction, but Mishchenko [Misc74] found a way to proceed and later Mishchenko-Fomenko [MiFo79] constructed a $C_{r}^{*}(G)$ bundle over $M$ and use this in place of the family of bundles indexed by the dual group, and then use this to the twisted elliptic operator. Then the twisted elliptic operator has its index in $K\left(C_{r}^{*}(G)\right)$ as desired. This is only the barest outline of a very complex construction in a very special case, but should provide some sense of the flavor of the subject.

For homology and cohomology of $C^{*}$-algebras the situation is more subtle, and as Connes has pointed out in [Con85b] and [Con94], cyclic cohomology and homology groups are the objects that one wants. However cyclic (co)homology does not work at all well for the original $C^{*}$-algebra; to obtain significant and useful results Connes argues that one has to replace the $C^{*}$-algebra of the groupoid by a suitable dense subalgebra of the $C^{*}$-algebra and use the cyclic (co)homology of that subalgebra instead. In the case of a compact manifold, the subalgebra consists of the $C^{\infty}$ functions and gives rise to DeRham theory; in the case of the group $C^{*}$-algebra of a discrete group, one could use the group algebra consisting of functions of compact support or an algebra of functions of suitably defined rapid descent at infinity or even those of finite support. Finally for the groupoid $M \times \Gamma$ generated by $C^{\infty}$ action of 
a discrete group on a compact manifold a suitable subalgebra might be the $C^{\infty}$ functions of compact support on $M \times \Gamma$. See Connes [Con86], Tsygan [Tsy83], Loday-Quillen [LoQu84] and Karoubi [Kar87]. Connes in [Con94] presents a general overview.

In the commutative case, a key ingredient for computations and applications of $K$-theory is the Chern character which is a map from $K$-theory to cohomology. This comes from calculating certain curvature forms associated to vector bundles, and associating to them their cohomology classes. Classically it is a rational isomorphism from $K$-cohomology to ordinary cohomology. A major goal in the non-commutative case is to prove the existence and key properties of a Chern character. One problem as noted already is that $K$-theory and cohomology theory live in different algebras. Cuntz in [Cun97] has taken on this challenge by defining a new kind of bivariate $K K$-theory but now defined for pairs of complete topological algebras, like the algebra of $C^{\infty}$ functions. He defines what he calls the $k k$-theory for such pairs, which he can characterize axiomatically. Then based on earlier joint work with Quillen [CuQu95a, CuQu95b, CuQu97], he is able to define a Chern charcter from his $k k$-theory to a bivariate periodic cyclic homology. The hope is that one can choose appropriately the subalgebras of $C^{*}$-algebras to insert into this $k k$-theory which are large enough so that they capture the $K K$-theory of the original algebras, but small enough so that they have interesting cyclic homology.

In light of this discussion of cyclic (co)homology, and the important roles that dense subalgebras of $C^{*}$ - often consisting of algebras of differentiable functions, suitably definedplay in the theory, it seems appropriate to have a concept and theory of another category of groupoids covering these objects, namely smooth groupoids or Lie groupoids. The definition almost makes itself: one wants a groupoid with the structure of say a $C^{\infty}$ manifold on $G$ and on the unit space, with all maps smooth and also such that the range and source maps are submersions - see [Hae84]. These notions are in fact quite venerable and can be traced back to Ehresmann [Ehr59]. Lie groupoids and their associated Lie algebroids play a large role in differential geometry that we will not go into here - see for instance [Mack87], [Mack05], [Wein87] and [Wein92]. Whenever a Lie group $H$ acts smoothly on a manifold $M$, the transformation group groupoid $M \times H$ is a Lie groupoid, and of course the foliation groupoid of a foliation is also such groupoid - see also [MoMr03]. As noted above the cyclic homology and cohomology of convolution algebras built on such groupoids carry significant geometric information.

One can formulate what it means for two Lie groupoids to be equivalent [MoMr03], and the definition is quite parallel to the notion of equivalence of topological groupoids described above. Étale Lie groupoids, where the range and source maps are local diffeomorphisms are of especial interest. Crainic and Moerdijk have established a theorem characterizing when a Lie groupoid is equivalent to an étale groupoid. Their result [CrMo00] is that the obvious necessary condition that the holonomy groups be discrete, is also sufficient. This result parallels the similar result for measured groupoids discussed in section 3, and involves a very general "slice theorem" for Lie groupoids. The authors also show that cyclic homologies of the 
convolution algebras of equivalent Lie groupoids are isomorphic - something one obviously wants to know, and which is very much in line with the general approach to groupoids that significant concepts and properties should be invariant under equivalence, similarity, or stable isomorphism appropriate to the category of groupoids with which one is dealing.

12. Borel Equivalence Relations and Groupoids. We now turn to a discussion of a fourth direction of research that was at least partially inspired by Mackey's notion of a virtual group. This concerns the study of equivalence relations in the Borel category, and as such is really a branch of descriptive set theory, and so lies on the boundary between logic and functional analysis. Alexander Kechris and Greg Hjorth have been leading workers in the field along with their co-workers. In this context one considers a standard Borel space $X$ and an equivalence relation $E$ on $X$ so $R$ is a subset of $X \times X$, and it is assumed to be a Borel subset. Following Mackey's lead, one should also extend this to include the natural the notion of a standard Borel groupoid that bears the same relation to standard Borel equivalence relations as groupoids to do equivalence relations. If one has a Polish group $H$ acting as a topological transformation on a Polish space $X$, then the transformation groupoid $G=X \times H$ is a prime candidate for such objects provided that the corresponding equivalence relation is a Borel subset of $X \times X$. There are no measures around as in the case of measured groupoids or measured equivalence relations, and results and definitions in the Borel category are phrased in terms of equations and relationships that now have to hold everywhere, not just almost everywhere.

The focus here, just as it has been in previous sections of this survey, will be on such equivalence relations which have countable orbits, and on Borel groupoids that are countable in the sense that the range and source maps are countable to one. This is equivalent to the orbits being countable - that is, the associated principal groupoid is a countable equivalence relation, and that the holonomy groups are all countable. One result from [FM77] comes over immediately and says that any such equivalence relation arises from the action of a countable group of Borel automorphisms of the space $X$. It also reasonable to ask about the structure of equivalence relations generated by more general groups, and Kechris has supplied a elegant answer [Ke92, Ke94]. He shows that if a locally compact (second countable as always) group acts on standard Borel space as a Borel transformation group, there is always complete lacunary section $S$ - a Borel subset which meets every orbit and so that there exists a neighborhood $U$ of the identity in $G$ such that $S U \cap S=S$. Such a set $S$ is automatically a complete transversal - that is, it meets each orbit in at most a countable set. He then goes on to show that X can be partitioned into $G$-invariant Borel sets $C$ and $Y$ such that the orbits of $G$ on $C$ are all countable, and that the equivalence relation defined by the action of $G$ on $Y$ is isomorphic to $R_{Z} \times \mathscr{I}$, where $Z$ is a complete lacunary section for the action of $G$ on $Y$ and $R_{Z}$ is the induced equivalence relation on $Z$ (which has countable orbits) and $\mathscr{I}$ is as before in the measured case, the Borel equivalence relation on the unit interval $I$ in which all points are equivalent - so the relation is just $I \times I$, but there are no measures here. 
In the case of general Borel equivalence relations, one, just as in the measure theoretic case, says that two relations $E$ and $F$ are (continuously) stably isomorphic if $E \times \mathscr{I}$ is isomorphic to $F \times \mathscr{I}$ where $\mathscr{I}$ is as above the relation on the unit interval with all points equivalent. Kechris's results then say that being stably isomorphic to a countable relation is the same as being generated by a Borel action of a locally compact group. Thus the equivalence relations generated by Borel actions of locally compact groups can be completely understood and classified by countable Borel equivalence relations.

From now on we stick with countable standard equivalence relations, and in at least one case below with a standard countable Borel groupoid. An essential point here is the introduction an order relation among such equivalence relations. One says that $E$ is Borel reducible to $F$ if and only if there is a Borel map $f$ from the unit space $X$ of $E$ into the unit space $Y$ of $F$ such that $(u, v) \in E$ if and only if $(f(u), f(v)) \in F$. This says that $f$ induces an injection or embedding of the "little space" of $E$ into the "little space" of $F$. One thinks of $E$ as being less than or equal to $F$ or that its classification problem of $E$ is less complex than that of $F$ and is written $E \leq_{B} F$. If also $F \leq_{B} E$, one says that $E$ and $F$ are bi-reducible written $E \sim_{B} F$. Just as in the measurable case for countable relations, one says that $E$ and $F$ are stably isomorphic if $E \times I_{\infty}$ is Borel isomorphic to $E \times I_{\infty}$. Proposition 2.6 of [DJK94] says that bireducibility is the same as stable isomorphism, and by Kechris's results above stable isomorphism for countable equivalence elations is the same as continuous stable isomorphism. Stable isomorphism is also in turn the same as $\left.E\right|_{A}$ Borel isomorphic to $\left.F\right|_{B}$ for Borel subsets $A$ and $B$ of the unit spaces of $E$ and $F$ respectively which are full in that they meet every orbit. It follows also that reducibility is a partial order relationship on stable isomorphism classes of countable Borel equivalence relations. Just as in the measurable case, the interest here is classification and study of these objects up to stable isomorphism.

Before moving on to a more detailed study of countable equivalence relations, it is instructive to look for moment to look higher up in the hierarchy of Borel equivalence relations above countable ones or those with a complete transversal. Of course it is easy to produce examples of Borel equivalence relations that do not have a complete transversal: one of the simplest examples is the equivalence relation on a countably infinite product of the circle group - the infinite dimensional torus $T^{\infty}$. The equivalence relation is defined by the action of the subgroup of those elements of $T^{\infty}$ all of whose coordinates are equal to 1 except for a finite set of indices. The equivalence relation makes equivalent those elements of the infinite dimensional torus whose coordinates agree off of a finite set - see [ $\mathrm{HjO0}]$. Indeed in this monograph, and in subsequent publications, Hjorth explores the hierarchy of equivalence relations above the countable ones and has a new approach to actions of Polish topological groups on Polish spaces.

First he defines what should be the next layer up above those relations Borel reducible to countable ones in the hierarchy - those Borel equivalence relations that are Borel reducible to one generated by a continuous action on a Polish space of a closed subgroup of a subgroup 
of $S_{\infty}$ the group of all permutations of a countably infinite set-call them permutation Borel relations. Then he defines two new types of group actions of a Polish group on a Polish space - stormy ones $[\mathrm{Hj} 05 \mathrm{~b}]$ and turbulent ones [Hj00] and [Hj02]. He then establishes two dichotomy theorems. His first dichotomy theorem is that an equivalence relation generated by an action of Polish group on a Polish space is either Borel reducible to a countable one or there is a continuous equivariant embedding of a stormy relation into it. (Note here that being Borel reducible to a countable relation is not the same as having a complete transversal in view of $[\mathrm{HjO5c]}$.) The second dichotomy theorem is that either the relation is Borel reducible to a permutation relation or there is a continuous equivariant embedding of a turbulent action into it. These are called dichotomy theorems based on the Effros-Glimm dichotomy theorem about group actions [Eff80].

13. Hyperfiniteness, Amenability, and Trees. The major thrust of what we will discuss consists of results that explore the nature of the order relationship among countable relations. First of all, one excludes the tame or smooth equivalence relations - that is the ones where there is a Borel selector - that is Borel subset of the unit space that meets each orbit in exactly one point - as the trivial case. Then it turns out that among the non-smooth relations there is a unique minimal element in this partial order-namely what is called $E_{0}$, which is the equivalence relation on $2^{N}$, the Cantor set of sequences of 0 's and 1 's, where two sequences are equivalent if they agree except on a finite set of indices. That this is a minimal element of the partial order is a result of the Borel Dichotomy Theorem of Harrington, Kechris and Louveau [HKL90] or actually in this simple case, it follows from the original Glimm- Effros dichotomy theorem [Eff80]. What is somewhat surprising is that there is also a maximal element of this partial order, usually written $E_{\infty}$. The unit space for this relation is all functions from the free group on two generators $F_{2}$, into $\{0,1\}$ so again a Cantor set. The equivalence relation is generated by the action of $F_{2}$ on this space by group translation on such functions. That this is maximal in the partial order follows from Proposition 1.8 of [DJK94].

The relation $E_{0}$ is clearly hyperfinite in that it is a union of an ascending sequence of relations with finite orbits. Just as in the measured case, it is known that this condition is equivalent to the equivalence relation being generated by a single transformation, and it is proved in [DJK94] that the non-smooth hyperfinite equivalence relations form a single stable isomorphism class. The isomorphism classes of Borel hyperfinite relations can also be enumerated in an elegant manner at least if one looks just at the aperiodic ones - that is those relations that have no finite orbits. (Note that finite orbits are washed out in stable isomorphism.) The non-smooth aperiodic Borel hyperfinite equivalence relations are classified up to isomorphism by the cardinality of the set of invariant ergodic probability measures that the set of units carries. This cardinality can be anything from zero up to $\aleph_{0}$ plus the power of the continuum, and there is a simple list of examples representing each type [DJK94, Theorem 9.1 and Corollary 9.3]. It is interesting to compare the classification here 
of non-smooth countable hyperfinite objects here and in the measured equivalence relation case. There one looked only at ergodic ones and the stable isomorphism classification is given by the Mackey range of the Radon-Nikodym cocycle. With the exception of the measure preserving case where the Radon-Nikodym cocycle is trivial and the Mackey range is the free transitive flow of the real line, stable isomorphism is the same as isomorphism, while in the measure preserving case, the stable isomorphism class has two isomorphism types corresponding to finite or infinite invariant measure. In the Borel case, there is no notion of ergodicity and there is one stable isomorphism class, which splits at least for aperiodic ones into a countable number of isomorphism types depending on how many ergodic probability measures there are.

Another difference is that in the measure equivalence relation case one knows that any the action of any amenable group locally compact generates a hyperfinite equivalence relation. In the Borel case no such general result is known, although hyperfiniteness of the equivalence relation can be proved for actions of a limited class of discrete amenable groups, for instance if $G$ is a finite extension of a finitely generated nilpotent group [JKL02, Corollary 1.20]. Of course, a theorem that the equivalence relation generated by the action of any discrete amenable group would settle matters in a very elegant manner, but that is unknown. Various notions of amenability analogous to Zimmer's definition in the measured groupoid case have been proposed, and there are number of interesting results but so far nothing definitive has emerged. One result of special interest is that is $G$ is a countable group and if the equivalence relation generated by the action of $G$ on the set of functions from $G$ to $\{0,1\}$ is hyperfinite, then $G$ is amenable [DJK94, Corollary 1.8]. This fact also shows that the maximal element of the partial order of stable isomorphism classes of countable equivalence relations discussed two paragraphs above is not hyperfinite. (Otherwise that partial ordering would be rather uninteresting!)

In the spirit of the question of to what extent an equivalence relation $E$ will code information about a freely acting group that generates $E$, it appears that Borel equivalence relations share with the measured equivalence relations the general observation that hyperfinte equivalence relations code very little information about the group except possibly for amenability, and so matters are rather unrigid. However when one turns attention to highly non-commutative groups, aspects of rigidity appear and such equivalence relations do encode significant information about the groups. In addition not much was known about the complexity of the partial order of stable isomorphism types of countable Borel equivalence relations until the paper of Adams and Kechris [AdKe00]. In fact only four examples of distinct types were known, but in [AdKe00] the authors use techniques from Zimmer rigidity theory to prove some remarkable results. They show in particular how to construct a countable Borel equivalence relation $E_{A}$ for each Borel subset $A$ of $[0,1]$ such that $A \rightarrow E_{A}$ is an order isomorphism form all Borel subsets of $[0,1]$ into the order structure of countable Borel equivalence relations up to stable isomorphism. This immediately showed that the order structure of this set is quite complex. 
In addition they showed that if $E_{n}$ is the countable Borel equivalence relation defined by the natural action of $G L_{n}(Z)$ on $T^{n}$, the $n$-dimensional torus, $n \geq 2$ then $n \rightarrow E_{n}$ is an order isomorphism. Thus $E_{n}$ up to stable isomorphism codes $n$, a result much weaker than what one had in the measured equivalence relation case.

Again in parallel with the measured equivalence relation case, the notion of a treeable equivalence relation can be defined. The definition is simply that one can assign in a Borel fashion the structure of a tree - a connected acyclic graph - to each equivalence class of the relation. If one can do this so that all graphs are locally finite trees, the relation is said to be locally finite treeable. It turns out there is no difference between these two notions as Gaboriau, Jackson, Kechris, and Louveau have shown that any treeable Borel equivalence relation is locally finitely treeable [JKL02, p. 48]. It also follows that treeability is an invariant under stable isomorphism and that if a free group on any number of generators acts freely on a space $X$, the equivalence relation generated is treeable [JKL02, p. 38-39]. Indeed more generally, any countable group which acts on a tree such that the stabilizer of each vertex is finite has the property that any free action on a Borel space generates a treeable relation [JKL02, p. 41]. A countable hyperfinite relation is treeable and any non-smooth treeable relation must be larger than $E_{0}$, the minimal countable non-smooth Borel equivalence. There is also a maximal treeable countable Borel equivalence relation in the partial order, namely the action of the free group on two generators $F_{2}$ on the free part of the action of $F_{2}$ on the set of all functions on $F_{2}$ to $\{0,1\}$, which in turn is less than the maximal countable Borel equivalence relation $E_{\infty}$ as the later is not treeable [JKL02, p. 61].

Just as for measured equivalence relations treeable Borel equivalence relations have a remarkable property with respect to homomorphisms which in fact also characterizes them. These facts, as we noted in section 7, were first discovered for Borel groupoids, and were then applied to the study of measured groupoids. Suppose $E$ is a countable standard Borel equivalence relation and that $G$ is a countable standard groupoid with a surjective Borel homomorphism $f$ of $G$ to $E$ with $f$ being countable to one and inducing a stable isomorphism of the equivalence relations. Then there is a lifting - that is there is a Borel homomorphism $g$ of $E$ into $G$ such that $f(g(u))=u$ for $u \in E$. This is a slight strengthening of the cocycle property of $[\mathrm{HjKe} 96]$; as stated there, the cocycle property corresponds to the existence of a lifting map on the special case when the groupoid $G$ is the groupoid $X \times H$ defined the action of a countable group $H$ on the unit space $X$ of $E$ so that $E$ is the principal groupoid (equivalence relation) associated to $X \times H$. The arguments in [HjKe96] can be used to show that this variant of the property is equivalent to the original cocycle property. Phrased in this manner the lifting property is very reminiscent of the lifting property of a homomorphism onto a free group.

This lifting property also implies that if $E$ is treeable, and if $H$ is any countable group which by an action on the unit space $X$ of $E$ generates, then there is an $F$ stably isomorphic to $E$ and a free action of $H$ on the unit space $Y$ of $F$ which generates $F$. This property also characterizes treeability [JKL02, p. 45]. Finally we note that this is one positive answer to 
the question left open in [FM77] about generating equivalence relations with free actions. It is left open as a question in [JKL02, p. 74] whether every countable Borel equivalence relation is stably isomorphic to one that is generated by a free action of some group - something that is definitely not the case as we have seen for measured equivalence relations. Simon Thomas in a December 2006 manuscript [Th06] has announced a counterexample to this.

14. Concluding Remarks. What we have tried to do here is, starting with Mackey's work in the 1960's on measured groupoids and virtual groups, to explore various themes over the following decades which we can trace back to Mackey's work. As indicated at the beginning of this article, the scope is vast and we have tried to focus on those developments that Mackey would have found most satisfying himself. One observation is that his influence was in a sense sequential in the four areas that we have discussed. The first area where his idea infiltrated was in measured groupoids and von Neumann algebras, beginning in the early to mid 1970's. The second area where they infiltrated was in topological groupoids, $C^{*}$-algebras, and noncommutative geometry in the mid to late 1970's. Mackey's ideas also infiltrated into the study of Lie groupoids in the mid 1980's and then finally his ideas infiltrated into the study of Borel equivalence relations as this subject developed as a part of descriptive set theory in the 1990's. A common theme is that it is possible to treat groupoids and discuss their properties in many respects in the same way that one does groups. Indeed the similarities are deeper than they appear at first glance. Thinking of groupoids or equivalence relations as virtual groups and likening them to ordinary subgroups is a constant theme. Another theme is how properties of a group inherit to properties of the virtual subgroup, and conversely, in the case when the virtual subgroup has an invariant measure and the group action is free, how properties of the virtual group propagate back to properties of the group.

\section{References}

[Ada88a] Adams, Scot. An equivalence relation that is not freely generated. Proc. Amer. Math. Soc. 102 (1988), no. 3, 565-566.

[Ada88b] Adams, Scot. Indecomposability of treed equivalence relations. Israel J. Math. 64 (1988), no. 3, 362-380.

[Ada90] Adams, Scot. Trees and amenable equivalence relations. Ergodic Theory Dynam. Systems 10 (1990), no. 1, 1-14.

[AEG94] Adams, Scot; Elliott, George A.; Giordano, Thierry. Amenable actions of groups. Trans. Amer. Math. Soc. 344 (1994), no. 2, 803-822.

[AdKe00] Adams, Scot; Kechris, Alexander S. Linear algebraic groups and countable Borel equivalence relations. J. Amer. Math. Soc. 13 (2000), no. 4, 909-943. 
[AdSp90] Adams, Scot; Spatzier, Ralf. Kazhdan groups, cocycles and trees. Amer. J. Math. 112 (1990), no. 2, 271-287.

[AkWa81] Akemann, Charles; Walter, Martin. Unbounded negative definite functions. Canad. J. Math. 33 (1981), no. 4, 862-871.

[AD97] Anantharaman-Delaroche, Claire. Classification des $C^{*}$-algèbres purement infinies nucléaires (d'après E. Kirchberg). Séminaire Bourbaki, Vol. 1995/96. Astérisque No. 241 (1997), Exp. No. 805, 3, 7-27.

[AD05] Anantharaman-Delaroche, Claire. Cohomology of property $T$ groupoids and applications. Ergodic Theory Dynam. Systems 25 (2005), no. 4, 977-1013.

[ADRe00] Anantharaman-Delaroche, C.; Renault, J. Amenable groupoids. With a foreword by Georges Skandalis and Appendix B by E. Germain. Monographies de L'Enseignement Mathématique, 36. L'Enseignement Mathématique, Geneva, 2000. 196 pp.

[ArWo69] Araki, Huzihiro; Woods, E.J. A classification of factors. Publ. Res. Inst. Math. Sci. Ser. A 4 1968/1969 51-130.

[Ati76] Atiyah, Michael F. Elliptic operators, discrete groups and von Neumann algebras. Colloque "Analyse et Topologie" en l'Honneur de Henri Cartan (Orsay, 1974), pp. 43-72. Asterisque, No. 32-33, Soc. Math. France, Paris, 1976.

[AtSi71] Atiyah, Michael F.; Singer, Isadore M. The index of elliptic operators. IV. Ann. of Math. (2) 93 (1971) 119-138.

[BC88a] Baum, Paul; Connes, Alain. Chern character for discrete groups. A fête of topology, 163-232, Academic Press, Boston, MA, 1988.

[BC88b] Baum, Paul; Connes, Alain. $K$-theory for discrete groups. Operator algebras and applications, Vol. 1, 1-20, London Math. Soc. Lecture Note Ser., 135, Cambridge Univ. Press, Cambridge, 1988.

[BC00] Baum, Paul; Connes, Alain. Geometric $K$-theory for Lie groups and foliations. Enseign. Math. (2) 46 (2000), no. 1-2, 3-42.

[BCH94] Baum, Paul; Connes, Alain; Higson, Nigel. Classifying space for proper actions and $K$-theory of group $C^{*}$-algebras. $C^{*}$-algebras: 1943-1993 (San Antonio, TX, 1993), 240-291, Contemp. Math., 167, Amer. Math. Soc., Providence, RI, 1994. 
[BaDo82] Baum, Paul; Douglas, Ronald. $K$ homology and index theory. Operator algebras and applications, Part I (Kingston, Ont., 1980), pp. 117-173, Proc. Sympos. Pure Math., 38, Amer. Math. Soc., Providence, R.I., 1982.

[BDF77] Brown, Lawrence; Douglas, Ronald; Fillmore, Peter. Extensions of $C^{*}$-algebras and $K$-homology. Ann. of Math. (2) 105 (1977), no. 2, 265-324.

[Bel68] Belinskaja, R. M. Partitionings of a Lebesgue space into trajectories which may be defined by ergodic automorphisms. Funkcional. Anal. i Prilozen. 2, no. 3 (1968), 4-16.

[Br87] Brown, Ronald. From groups to groupoids: a brief survey. Bull. London Math. Soc. 19 (1987), no. 2, 113-134.

[BuMo99] Burger, M.; Monod, N. Bounded cohomology of lattices in higher rank Lie groups. J. Eur. Math. Soc. (JEMS) 1 (1999), no. 2, 199-235.

[BuMo02] Burger, M.; Monod, N. Continuous bounded cohomology and applications to rigidity theory. Geom. Funct. Anal. 12 (2002), no. 2, 219-280.

[CC00] Candel, Alberto; Conlon, Lawrence. Foliations. I. Graduate Studies in Mathematics, 23. American Mathematical Society, Providence, RI, 2000. xiv+402 pp.

[CC03] Candel, Alberto; Conlon, Lawrence. Foliations. II. Graduate Studies in Mathematics, 60. American Mathematical Society, Providence, RI, 2003. xiv +545 pp.

[ChGr86] Cheeger, Jeff; Gromov, Mikhael. $L^{2}$-cohomology and group cohomology. Topology 25 (1986), no. 2, 189-215.

[CCJJV01] Cherix, Pierre-Alain; Cowling, Michael; Jolissaint, Paul; Julg, Pierre; Valette, Alain. Groups with the Haagerup property. Gromov's a-T-menability. Progress in Mathematics, 197. Birkhuser Verlag, Basel, 2001. viii+126 pp.

[Ch83] Choda, Marie. Group factors of the Haagerup type. Proc. Japan Acad. Ser. A Math. Sci. 59 (1983), no. 5, 174-177.

[Con73] Connes, Alain. Une classification des facteurs de type III. Ann. Sci. École Norm. Sup. (4) 6 (1973), 133-252.

[Con76] Connes, Alain. Classification of injective factors. Cases $I I_{1}, I I_{\infty}, I I I_{\lambda}, \lambda \neq 1$. Ann. of Math. (2) 104 (1976), no. 1, 73-115. 
[Con79] Connes, Alain. Sur la théorie non commutative de l'intégration. Algèbres d'opérateurs (Sém., Les Plans-sur-Bex, 1978), pp. 19-143, Lecture Notes in Math., 725, Springer, Berlin, 1979.

[Con80] Connes, Alain. A factor of type $I I_{1}$ with countable fundamental group. J. Operator Theory 4 (1980), no. 1, 151-153.

[Con81] Connes, Alain. An analogue of the Thom isomorphism for crossed products of a $C^{*}$-algebra by an action of $R$. Adv. in Math. 39 (1981), no. 1, 31-55.

[Con82] Connes, Alain. A survey of foliations and operator algebras. Operator algebras and applications, Part I (Kingston, Ont., 1980), pp. 521-628, Proc. Sympos. Pure Math., 38, Amer. Math. Soc., Providence, R.I., 1982.

[Con85a] Connes, Alain. Factors of type $I I I_{1}$, property $L_{\lambda}^{\prime}$ and closure of inner automorphisms. J. Operator Theory 14 (1985), no. 1, 189-211.

[Con85b] Connes, Alain. Noncommutative differential geometry. Inst. Hautes Études Sci. Publ. Math. No. 62 (1985), 257-360.

[Con86] Connes, Alain. Cyclic cohomology and the transverse fundamental class of a foliation. Geometric methods in operator algebras (Kyoto, 1983), 52-144, Pitman Res. Notes Math. Ser., 123, Longman Sci. Tech., Harlow, 1986.

[Con94] Connes, Alain. Noncommutative geometry. Academic Press, Inc., San Diego, CA, 1994. xiv+661 pp.

[CFW81] Connes, Alain; Feldman, Jacob; Weiss, Benjamin. An amenable equivalence relation is generated by a single transformation. Ergodic Theory Dynamical Systems 1 (1981), no. 4, 431-450.

[CoJo82] Connes, Alain; Jones, Vaughan. A $I I_{1}$ factor with two nonconjugate Cartan subalgebras. Bull. Amer. Math. Soc. (N.S.) 6 (1982), no. 2, 211-212.

[CoJo85] Connes, Alain; Jones, Vaughan. Property $(T)$ for von Neumann algebras. Bull. London Math. Soc. 17 (1985), no. 1, 57-62.

[CoKr77] Connes, Alain; Krieger, Wolfgang. Measure space automorphisms, the normalizers of their full groups, and approximate finiteness. J. Functional Analysis 24 (1977), no. 4, 336-352.

[CoMo90] Connes, Alain; Moscovici, Henri. Cyclic cohomology, the Novikov conjecture and hyperbolic groups. Topology 29 (1990), no. 3, 345-388. 
[CoSk84] Connes, Alain; Skandalis, Georges. The longitudinal index theorem for foliations. Publ. Res. Inst. Math. Sci. 20 (1984), no. 6, 1139-1183.

[CoTa77] Connes, Alain; Takesaki, Masamichi. The flow of weights on factors of type III. Tôhoku Math. J. (2) 29 (1977), no. 4, 473-575.

[CoWe80] Connes, Alain; Weiss, Benjamin. Property $(T)$ and asymptotically invariant sequences. Israel J. Math. 37 (1980), no. 3, 209-210.

[CowZim89] Cowling, Michael; Zimmer, Robert J. Actions of lattices in $S p(1, n)$. Ergodic Theory Dynam. Systems 9 (1989), no. 2, 221-237.

[CrMo00] Crainic, Marius; Moerdijk, Ieke. A homology theory for étale groupoids. J. Reine Angew. Math. 521 (2000), 25-46.

[CrMo01] Crainic, Marius; Moerdijk, Ieke. Foliation groupoids and their cyclic homology. Adv. Math. 157 (2001), no. 2, 177-197.

[Cun81] Cuntz, Joachim. A class of $C^{*}$-algebras and topological Markov chains. II. Reducible chains and the Ext-functor for $C^{*}$-algebras. Invent. Math. 63 (1981), no. $1,25-40$.

[Cun83] Cuntz, Joachim. K-theoretic amenability for discrete groups. J. Reine Angew. Math. 344 (1983), 180-195.

[Cun97] Cuntz, Joachim. Bivariante $K$-Theorie für lokalkonvexe Algebren und der Chern-Connes-Charakter. Doc. Math. 2 (1997), 139-182

[CuKr80] Cuntz, Joachim; Krieger, Wolfgang. A class of $C^{*}$-algebras and topological Markov chains. Invent. Math. 56 (1980), no. 3, 251-268.

[CuQu95a] Cuntz, Joachim; Quillen Daniel. Operators on noncommutative differential forms and cyclic homology. Geometry, topology, \& physics, 77-111, Conf. Proc. Lecture Notes Geom. Topology, IV, Int. Press, Cambridge, MA, 1995.

[CuQu95b] Cuntz, Joachim; Quillen Daniel. Cyclic homology and nonsingularity. J. Amer. Math. Soc. 8 (1995), no. 2, 373-442.

[CuQu97] Cuntz, Joachim; Quillen, Daniel. Excision in bivariant periodic cyclic cohomology. Invent. Math. 127 (1997), no. 1, 67-98.

[DJK94] Dougherty, R.; Jackson, S.; Kechris, A.S. The structure of hyperfinite Borel equivalence relations. Trans. Amer. Math. Soc. 341 (1994), no. 1, 193-225. 
[Dou80] Douglas, Ronald. $C^{*}$-algebra extensions and $K$-homology. Annals of Mathematics Studies, 95. Princeton University Press, Princeton, N.J.; University of Tokyo Press, Tokyo, 1980. vii+84 pp.

[Dye59] Dye, Henry. On groups of measure preserving transformation. I. Amer. J. Math. 81 (1959) 119-159.

[Dye63] Dye, Henry. On groups of measure preserving transformations. II. Amer. J. Math. 85 (1963) 551-576.

[Eff80] Effros, Edward. Polish transformation groups and classification problems. General topology and modern analysis (Proc. Conf., Univ. California, Riverside, Calif., 1980), pp. 217-227, Academic Press, New York-London, 1981

[EfHa67] Effros, Edward; Hahn, Frank. Locally compact transformation groups and $C^{*}$ algebras. Memoirs of the American Mathematical Society, No. 75 American Mathematical Society, Providence, R.I. 196792 pp.

[Ehr59] Ehresmann, Charles. Catégories topologiques et catégories différentiables. (French) 1959 Colloque Géom. Diff. Globale (Bruxelles, 1958) pp. 137-150 Centre Belge Rech. Math., Louvain

[ExRe06] Exel, Ruy; Renault, Jean. $A F$-algebras and the tail-equivalence relation on Bratteli diagrams. Proc. Amer. Math. Soc. 134 (2006), no. 1, 193-206

[FHM78] Feldman, Jacob; Hahn, Peter; Moore, Calvin C. Orbit structure and countable sections for actions of continuous groups. Adv. in Math. 28 (1978), no. 3, 186230.

[FM77] Feldman, Jacob; Moore, Calvin C. Ergodic equivalence relations, cohomology, and von Neumann algebras. I \& II. Trans. Amer. Math. Soc. 234 (1977), no. 2, 289-324 \& 325-359.

[FeRa85] Feldman, Jacob; Ramsay, Arlan. Countable sections for free actions of groups. Adv. in Math. 55 (1985), no. 3, 224-227.

[Fur99a] Furman, Alex. Gromov's measure equivalence and rigidity of higher rank lattices. Ann. of Math. (2) 150 (1999), no. 3, 1059-1081.

[Fur99b] Furman, Alex. Orbit equivalence rigidity. Ann. of Math. (2) 150 (1999), no. 3, 1083-1108. 
[Gab00] Gaboriau, Damien. Coût des relations d'équivalence et des groupes. (French) Cost of equivalence relations and of groups. Invent. Math. 139 (2000), no. 1, $41-98$.

[Gab02] Gaboriau, Damien. Invariants $l^{2}$ de relations d'équivalence et de groupes. Publ. Math. Inst. Hautes Études Sci. No. 95 (2002), 93-150.

[GaPo05] Gaboriau, Damien; Popa, Sorin. An uncountable family of nonorbit equivalent actions of $\mathbb{F}_{n}$. J. Amer. Math. Soc. 18 (2005), no. 3, 547-559.

[GeGo88] Gefter, Sergey; Golodets, Valentin. Fundamental groups for ergodic actions and actions with unit fundamental groups. Publ. Res. Inst. Math. Sci. 24 (1988), no. $6,821-847$.

[GoRo79] Gootman, Elliot; Rosenberg, Jonathan. The structure of crossed product $C^{*}$ algebras: a proof of the generalized Effros-Hahn conjecture. Invent. Math. 52 (1979), no. 3, 283-298.

[Gro88] Gromov, Mikhael. Rigid transformations groups. Géométrie différentielle (Paris, 1986), 65-139, Travaux en Cours, 33, Hermann, Paris, 1988.

[Gro93] Gromov, Mikhael. Asymptotic invariants of infinite groups. Geometric group theory, Vol. 2 (Sussex, 1991), 1-295, London Math. Soc. Lecture Note Ser., 182, Cambridge Univ. Press, Cambridge, 1993.

[Haa79] Haagerup, Uffe. An example of a nonnuclear $C^{*}$-algebra, which has the metric approximation property. Invent. Math. 50 (1978/79), no. 3, 279-293.

[Haa87] Haagerup, Uffe. Connes' bicentralizer problem and uniqueness of the injective factor of type $I I I_{1}$. Acta Math. 158 (1987), no. 1-2, 95-148.

[Hae79] Haefliger, André. Differential cohomology. Differential topology (Varenna, 1976), pp. 19-70, Liguori, Naples, 1979.

[Hae84] Haefliger, André. Groupoïdes d'holonomie et classifiants. Astérisque No. 116 (1984), 70-97.

[Hae01] Haefliger, André. Groupoids and foliations. Groupoids in analysis, geometry, and physics (Boulder, CO, 1999), 83-100, Contemp. Math., 282, Amer. Math. Soc., Providence, RI, 2001.

[Hah78a] Hahn, Peter. Haar measure for measure groupoids. Trans. Amer. Math. Soc. 242 (1978), 1-33. 
[Hah78b] Hahn, Peter. The regular representations of measure groupoids. Trans. Amer. Math. Soc. 242 (1978), 35-72.

[HKL90] Harrington, Leo; Kechris, Alexander; Louveau, Alain. A Glimm-Effros dichotomy for Borel equivalence relations. J. Amer. Math. Soc. 3 (1990), no. 4, 903-928.

[HiKa97] Higson, Nigel; Kasparov, Gennadi. Operator $K$-theory for groups which act properly and isometrically on Hilbert space. Electron. Res. Announc. Amer. Math. Soc. 3 (1997), 131-142.

[HiKa01] Higson, Nigel; Kasparov, Gennadi. E-theory and $K K$-theory for groups which act properly and isometrically on Hilbert space. Invent. Math. 144 (2001), no. $1,23-74$.

[HLS02] Higson, Nigel; Lafforgue, Vincent; Skandalis, Georges. Counterexamples to the Baum-Connes conjecture. Geom. Funct. Anal. 12 (2002), no. 2, 330-354.

[Hj00] Hjorth, Greg. Classification and orbit equivalence relations. Mathematical Surveys and Monographs, 75. American Mathematical Society, Providence, RI, 2000. xviii+195 pp.

[Hj02] Hjorth, Greg. A dichotomy theorem for turbulence. J. Symbolic Logic 67 (2002), no. $4,1520-1540$.

[Hj05a] Hjorth, Greg. A converse to Dye's theorem. Trans. Amer. Math. Soc. 357 (2005), no. 8, 3083-3103.

[Hj05b] Hjorth, Greg. A dichotomy theorem for being essentially countable. Logic and its applications, 109-127, Contemp. Math., 380, Amer. Math. Soc., Providence, RI, 2005.

[Hj05c] Hjorth, Greg. Bi-Borel reducibility of essentially countable Borel equivalence relations. J. Symbolic Logic 70 (2005), no. 3, 979-992.

[Hj06] Hjorth, Greg. A lemma for cost attained. Ann. Pure Appl. Logic 143 (2006), no. 1-3, 87-102.

[HjKe96] Hjorth, Greg; Kechris, Alexander S. Borel equivalence relations and classifications of countable models. Ann. Pure Appl. Logic 82 (1996), no. 3, 221-272.

[HjKe97] Hjorth, Greg; Kechris, Alexander S. New dichotomies for Borel equivalence relations. Bull. Symbolic Logic 3 (1997), no. 3, 329-346. 
[JKL02] Jackson, S.; Kechris, A. S.; Louveau, A. Countable Borel equivalence relations. J. Math. Log. 2 (2002), no. 1, 1-80.

[Jo05] Jolissaint, Paul. The Haagerup property for measure-preserving standard equivalence relations. Ergodic Theory Dynam. Systems 25 (2005), no. 1, 161-174.

[Kar87] Karoubi, Max. Homologie cyclique et K-théorie. Astérisque No. 149 (1987), 147 pp.

[Ka75] Kasparov, Gennadi. Topological invariants of elliptic operators. I. $K$-homology. Izv. Akad. Nauk SSSR Ser. Mat. 39 (1975), no. 4, 796-838

[Ka80] Kasparov, Gennadi. The operator $K$-functor and extensions of $C^{*}$-algebras. (Russian) Izv. Akad. Nauk SSSR Ser. Mat. 44 (1980), no. 3, 571-636, 719.

[Ka88] Kasparov, Gennadi. Equivariant $K K$-theory and the Novikov conjecture. Invent. Math. 91 (1988), no. 1, 147-201.

[Kaz67] Kazhdan, David. On the connection of the dual space of a group with the structure of its closed subgroups. Funkcional. Anal. i Priložen. 1 (1967) 71-74.

[Ke92] Kechris, Alexander S. Countable sections for locally compact group actions. Ergodic Theory Dynam. Systems 12 (1992), no. 2, 283-295.

[Ke94] Kechris, Alexander S. Countable sections for locally compact group actions. II. Proc. Amer. Math. Soc. 120 (1994), no. 1, 241-247.

[KeLo97] Kechris, Alexander S.; Louveau, Alain. The classification of hypersmooth Borel equivalence relations. J. Amer. Math. Soc. 10 (1997), no. 1, 215-242.

[Kr69] Krieger, Wolfgang. On non-singular transformations of a measure space. I, II. Z. Wahrscheinlichkeitstheorie und Verw. Gebiete 11 (1969), 83-97; ibid. 111969 98-119.

[Kr70] Krieger, Wolfgang. On constructing non-isomorphic hyperfinite factors of type III. J. Functional Analysis 6 (1970) 97-109.

[Kr71] Krieger, Wolfgang. On a class of hyperfinite factors that arise from nullrecurrent Markov chains. J. Functional Analysis 7 (1971) 27-42.

[Kr72] Krieger, Wolfgang. On the infinite product construction of non-singular transformations of a measure space. Invent. Math. 15 (1972), 144-163.

[Kr76] Krieger, Wolfgang. On ergodic flows and the isomorphism of factors. Math. Ann. 223 (1976), no. 1, 19-70. 
[Kum86] Kumjian, Alexander. On $C^{*}$-diagonals. Canad. J. Math. 38 (1986), no. 4, 9691008.

[Kum88] Kumjian, Alexander. On equivariant sheaf cohomology and elementary $C^{*}$ bundles. J. Operator Theory 20 (1988), no. 2, 207-240.

[KMRW98] Kumjian, Alexander; Muhly, Paul S.; Renault, Jean N.; Williams, Dana P. The Brauer group of a locally compact groupoid. Amer. J. Math. 120 (1998), no. 5, 901-954.

[KPRR97] Kumjian, Alexander; Pask, David; Raeburn, Iain; Renault, Jean. Graphs, groupoids, and Cuntz-Krieger algebras. J. Funct. Anal. 144 (1997), no. 2, 505541.

[KPR98] Kumjian, Alexander; Pask, David; Raeburn, Iain. Cuntz-Krieger algebras of directed graphs. Pacific J. Math. 184 (1998), no. 1, 161-174.

[Laf02] Lafforgue, Vincent. $K$-théorie bivariante pour les algèbres de Banach et conjecture de Baum-Connes. Invent. Math. 149 (2002), no. 1, 1-95.

[Lev95] Levitt, Gilbert. On the cost of generating an equivalence relation. Ergodic Theory Dynam. Systems 15 (1995), no. 6, 1173-1181.

[LoQu84] Loday, Jean-Louis; Quillen, Daniel. Cyclic homology and the Lie algebra homology of matrices. Comment. Math. Helv. 59 (1984), no. 4, 569-591.

[Lus72] Lusztig, George. Novikov's higher signature and families of elliptic operators. J. Differential Geometry 7 (1972), 229-256.

[Mack87] Mackenzie, Kirill. Lie groupoids and Lie algebroids in differential geometry. London Mathematical Society Lecture Note Series, 124. Cambridge University Press, Cambridge, 1987. xvi+327 pp.

[Mack05] Mackenzie, Kirill. General theory of Lie groupoids and Lie algebroids. London Mathematical Society Lecture Note Series, 213. Cambridge University Press, Cambridge, 2005. xxxviii+501 pp.

[Ma49] Mackey, George W. Imprimitivity for representations of locally compact groups. I. Proc. Nat. Acad. Sci. U. S. A. 35, (1949). 537-545.

[Ma51] Mackey, George W. On induced representations of groups. Amer. J. Math. 73, (1951). 576-592. 
[Ma57] Mackey, George W. Borel structure in groups and their duals. Trans. Amer. Math. Soc. 85 (1957), 134-165.

[Ma58] Mackey, George W. Unitary representations of group extensions. I. Acta Math. 99 (1958) 265-311.

[Ma63a] Mackey, George W. Infinite-dimensional group representations. Bull. Amer. Math. Soc. 69 (1963) 628-686.

[Ma63b] Mackey, George W. Ergodic theory, group theory, and differential geometry. Proc. Nat. Acad. Sci. U.S.A. 50 (1963) 1184-1191.

[Ma66] Mackey, George W. Ergodic theory and virtual groups. Math. Ann. 166 (1966) 187-207.

[MiYu02] Mineyev, Igor; Yu, Guoliang. The Baum-Connes conjecture for hyperbolic groups. Invent. Math. 149 (2002), no. 1, 97-122.

[Misc74] Mishchenko, Alexandr. Infinite-dimensional representations of discrete groups, and higher signatures. Izv. Akad. Nauk SSSR Ser. Mat. 38 (1974), 81-106.

[MiFo79] Mishchenko, Alexandr; Fomenko, Anatoly. The index of elliptic operators over $C^{*}$-algebras. Izv. Akad. Nauk SSSR Ser. Mat. 43 (1979), no. 4, 831-859, 967.

[Moer95] Moerdijk, Ieke. Classifying spaces and classifying topoi. Lecture Notes in Mathematics, 1616. Springer-Verlag, Berlin, 1995. vi+94 pp.

[Moer01] Moerdijk, Ieke. Étale groupoids, derived categories, and operations. Groupoids in analysis, geometry, and physics (Boulder, CO, 1999), 101-114, Contemp. Math., 282, Amer. Math. Soc., Providence, RI, 2001.

[MoMr03] Moerdijk, Ieke; Mrčun, Janos. Introduction to foliations and Lie groupoids. Cambridge Studies in Advanced Mathematics, 91. Cambridge University Press, Cambridge, 2003. $\mathrm{x}+173 \mathrm{pp}$.

[Mon01] Monod, Nicolas. Continuous bounded cohomology of locally compact groups. Lecture Notes in Mathematics, 1758. Springer-Verlag, Berlin, 2001. x+214 pp.

[Mon06] Monod, Nicolas. Superrigidity for irreducible lattices and geometric splitting. J. Amer. Math. Soc. 19 (2006), no. 4, 781-814

[MoSh04] Monod, Nicolas; Shalom, Yehuda. Cocycle superrigidity and bounded cohomology for negatively curved spaces. J. Differential Geom. 67 (2004), no. 3, $395-455$. 
[MoSh06] Monod, Nicolas; Shalom, Yehuda. Orbit equivalence rigidity and bounded cohomology. Ann. of Math. (2) 164 (2006), no. 3, 825-878.

[Moore76] Moore, Calvin C. Group extensions and cohomology for locally compact groups. III \& IV. Trans. Amer. Math. Soc. 221 (1976), no. 1, 1-33 \& 35-58.

[MoSc06] Moore, Calvin C.; Schochet, Claude. Global analysis on foliated spaces. Second edition. Mathematical Sciences Research Institute Publications, 9. Cambridge University Press, New York, 2006. xiv+293 pp.

[MRW87] Muhly, Paul S.; Renault, Jean N.; Williams, Dana P. Equivalence and isomorphism for groupoid $C^{*}$-algebras. J. Operator Theory 17 (1987), no. 1, 3-22.

[OrWe80] Ornstein, Donald; Weiss, Benjamin. Ergodic theory of amenable group actions. I. The Rohlin lemma. Bull. Amer. Math. Soc. (N.S.) 2 (1980), no. 1, 161-164.

[Popa06a] Popa, Sorin. On a class of type $I I_{1}$ factors with Betti numbers invariants. Ann. of Math. (2) 163 (2006), no. 3, 809-899.

[Popa06b] Popa, Sorin. Some computations of 1-cohomology groups and construction of non-orbit-equivalent actions. J. Inst. Math. Jussieu 5 (2006), no. 2, 309-332.

[Popa06c] Popa, Sorin. Strong rigidity of $I I_{1}$ factors arising from malleable actions of $w$-rigid groups. I \& II. Invent. Math. 165 (2006), no. 2, 369-408 \& 409-451.

[Ram71] Ramsay, Arlan. Virtual groups and group actions. Advances in Math. 6 (1971), 253-322.

[Ram76] Ramsay, Arlan. Nontransitive quasi-orbits in Mackey's analysis of group extensions. Acta Math. 137 (1976), no. 1, 17-48.

[Ram80] Ramsay, Arlan. Subobjects of virtual groups. Pacific J. Math. 87 (1980), no. 2, $389-454$.

[Ram82] Ramsay, Arlan. Topologies on measured groupoids. J. Funct. Anal. 47 (1982), no. $3,314-343$.

[Ram85] Ramsay, Arlan. Measurable group actions are essentially Borel actions. Israel J. Math. 51 (1985), no. 4, 339-346.

[Ram91] Ramsay, Arlan. Local product structure for group actions. Ergodic Theory Dynam. Systems 11 (1991), no. 1, 209-217.

[Ram97] Ramsay, Arlan. Lacunary sections for locally compact groupoids. Ergodic Theory Dynam. Systems 17 (1997), no. 4, 933-940. 
[RaRe01] Ramsay, Arlan; Renault, Jean (editors). Groupoids in analysis, geometry, and physics. Papers from the AMS-IMS-SIAM Joint Summer Research Conference held at the University of Colorado, Boulder, CO, June 20-24, 1999. Contemporary Mathematics, 282. American Mathematical Society, Providence, RI, 2001. xii+192 pp.

[Ren80] Renault, Jean. A groupoid approach to $C^{*}$-algebras. Lecture Notes in Mathematics, 793. Springer, Berlin, 1980. ii+160 pp.

[Ren82] Renault, Jean. $C^{*}$-algebras of groupoids and foliations. Operator algebras and applications, Part I (Kingston, Ont., 1980), pp. 339-350, Proc. Sympos. Pure Math., 38, Amer. Math. Soc., Providence, R.I., 1982.

[Ren91] Renault, Jean. The ideal structure of groupoid crossed product $C^{*}$-algebras. With an appendix by Georges Skandalis. J. Operator Theory 25 (1991), no. 1, $3-36$.

[Ren00] Renault, Jean. Cuntz-like algebras. Operator theoretical methods (Timişoara, 1998), 371-386, Theta Found., Bucharest, 2000.

[Ros83] Rosenberg, Jonathan. $C^{*}$-algebras, positive scalar curvature, and the Novikov conjecture. Inst. Hautes Études Sci. Publ. Math. No. 58 (1983) 197-212.

[Ros86] Rosenberg, Jonathan. $C^{*}$-algebras, positive scalar curvature and the Novikov conjecture. II. Geometric methods in operator algebras (Kyoto, 1983), 341-374, Pitman Res. Notes Math. Ser., 123, Longman Sci. Tech., Harlow, 1986.

[Ser81] Series, Caroline. An application of groupoid cohomology. Pacific J. Math. 92 (1981), no. 2, 415-432.

[Sh05] Shalom, Yehuda. Measurable group theory. European Congress of Mathematics, 391-423, Eur. Math. Soc., Zürich, 2005.

[Th06] Thomas, Simon. Popa superrigidity and countable Borel equivalence relations (downloaded from http://www.math.rutgers.edu/ sthomas/papers.html).

[Tsy83] Tsygan, Boris. Homology of matrix Lie algebras over rings and the Hochschild homology. (Russian) Uspekhi Mat. Nauk 38 (1983), no. 2(230), 217-218.

[Tu99a] Tu, Jean-Loius. La conjecture de Novikov pour les feuilletages hyperboliques. $K$-Theory 16 (1999), no. 2, 129-184.

[Tu99b] Tu, Jean-Louis. La conjecture de Baum-Connes pour les feuilletages moyennables. $K$-Theory 17 (1999), no. 3, 215-264. 
[Tu00] Tu, Jean-Louis. The Baum-Connes conjecture for groupoids. $C^{*}$-algebras (Münster, 1999), 227-242, Springer, Berlin, 2000.

[Tu04] Tu, Jean-Louis. Non-Hausdorff groupoids, proper actions and $K$-theory. Doc. Math. 9 (2004), 565-597.

[Tu06] Tu, Jean-Louis. Groupoid cohomology and extensions. Trans. Amer. Math. Soc. 358 (2006), no. 11, 4721-4747.

[Ver68] Vershik, Anatoly. A theorem on the lacunary isomorphism of monotonic sequences of partitionings. Funkcional. Anal. i Prilozen. 2 (1968), no. 3, 17-21.

[Vo96] Voiculescu, Dan. The analogues of entropy and of Fisher's information measure in free probability theory. III. The absence of Cartan subalgebras. Geom. Funct. Anal. 6 (1996), no. 1, 172-199.

[Wein87] Weinstein, Alan. Symplectic groupoids and Poisson manifolds. Bull. Amer. Math. Soc. (N.S.) 16 (1987), no. 1, 101-104.

[Wein92] Weinstein, Alan. Lagrangian mechanics and groupoids. Mechanics day (Waterloo, ON, 1992), 207-231, Fields Inst. Commun., 7, Amer. Math. Soc., Providence, RI, 1996.

[Wein01] Weinstein, Alan. Groupoids: unifying internal and external symmetry. A tour through some examples. Groupoids in analysis, geometry, and physics (Boulder, CO, 1999), 1-19, Contemp. Math., 282, Amer. Math. Soc., Providence, RI, 2001.

[Wes69] Westman, Joel. Cohomology for ergodic groupoids. Trans. Amer. Math. Soc. 146 (1969) 465-471.

[Wes71] Westman, Joel. Cohomology for the ergodic actions of countable groups. Proc. Amer. Math. Soc. 30 (1971) 318-320.

[Will01] Williams, Dana. A primer for the Brauer group of a groupoid. Groupoids in analysis, geometry, and physics (Boulder, CO, 1999), 21-34, Contemp. Math., 282, Amer. Math. Soc., Providence, RI, 2001

[Wi83] Winkelnkemper, H.E. The graph of a foliation. Ann. Global Anal. Geom. 1 (1983), no. 3, 51-75.

[Zim77] Zimmer, Robert J. Hyperfinite factors and amenable ergodic actions. Invent. Math. 41 (1977), no. 1, 23-31. 
[Zim78a] Zimmer, Robert J. Amenable ergodic group actions and an application to Poisson boundaries of random walks. J. Functional Analysis 27 (1978), no. 3, 350372 .

[Zim78b] Zimmer, Robert J. Induced and amenable ergodic actions of Lie groups. Ann. Sci. École Norm. Sup. (4) 11 (1978), no. 3, 407-428.

[Zim80] Zimmer, Robert J. Strong rigidity for ergodic actions of semi-simple Lie groups. Ann. of Math. (2) 112 (1980), no. 3, 511-529.

[Zim81] Zimmer, Robert J. On the cohomology of ergodic actions of semisimple Lie groups and discrete subgroups. Amer. J. Math. 103 (1981), no. 5, 937-951.

[Zim84] Zimmer, Robert J. Ergodic theory and semisimple groups. Monographs in Mathematics, 81. Birkhäuser Verlag, Basel, 1984. x+209 pp. 